\title{
A Recombinant DDAH-1 Based Biotherapeutics to Pharmacologically Lower ADMA, thus Improving Post Ischemic Cardiac Function and Cardiomyocyte Mitochondrial Activity
}

Young Lee, Jaipal Singh, Susan R. Scott, Bradley Ellis, Pinar Zorlutuna and Meijing Wang

Indiana Center for Biomedical Innovation, Indianapolis, IN (YL, JS), Indiana University School of Medicine, Indianapolis, IN (JS), Department of Surgery, Indiana University, School of Medicine, Indianapolis, IN (SS, MW), Bioengineering Graduate Program, University of Notre Dame, Notre Dame, IN (BE, PZ), Aerospace and Mechanical Engineering Department, University of Notre Dame, Notre Dame, IN (PZ), Vasculonics LLC, Indianapolis, IN (JS)

Running Title: Lowering of ADMA by Pharmacological DDAH-1 in Heart

Correspondence: Meijing Wang, MD

950 W. Walnut Street, R2 E319

Indianapolis, Indiana 46202

meiwang@iupui.edu

$317-274-0827$ 
Number of Text Pages: 39

Number of Tables: 0

Number of Figures: 8

Number of Supplemental Figures: 3

Number of References: 48

\title{
Number of Words
}

Abstract: 248

Introduction: 620

Discussion: 1022

\begin{abstract}
Abbreviation: ADMA, asymmetric dimethyl arginine; DDAH, dimethylarginine dimethylaminohydrolase; hiPSC, human induced pluripotent stem cell; iCM, induced pluripotent stem cell derived cardiomyocytes; I/R, ischemia-reperfusion injury; LV, left ventricle; LVDP, left ventricular developed pressure; mPTP, mitochondrial permeability transition pore; NOS, nitric oxide synthase; OCR, oxygen consumption rate; OPA, ortho-phthaldialdehyde; RLU, relative light units; ROCK, rho-associated, coiled-coil containing protein kinase; ROS, reactive oxygen species; TTC, 2,3,5triphenyltetrazolium chloride.
\end{abstract}


Molecular Pharmacology Fast Forward. Published on January 18, 2022 as DOI: 10.1124/molpharm.121.000394

This article has not been copyedited and formatted. The final version may differ from this version.

\section{Abstract}

High serum levels of asymmetric dimethyl arginine (ADMA) are associated with cardiovascular disease and mortality. Pharmacological agents to specifically lower ADMA, and their potential impact on cardiovascular complications are not known. In this study, we aimed to investigate the effect of specific lowering of ADMA on myocardial response to ischemia-reperfusion injury $(\mathrm{I} / \mathrm{R})$, and direct effects on cardiomyocyte function. Effects of recombinant DDAH-1 (rDDAH-1) on I/R injury were determined using isolated mouse heart preparation. Respiration capacity and mitochondrial reactive oxygen species (ROS) generation were determined on mouse cardiomyocytes. Our results show that lowering ADMA by rDDAH-1 treatment resulted in improved recovery of cardiac function and reduction in myocardial infarct size in response to I/R injury (control $22.24 \pm 4.60 \%$ vs rDDAH-1 $15.90 \pm 4.23 \%, \mathrm{p}<0.01$ ) to mouse heart. In mouse cardiomyocytes, rDDAH-1 treatment improved ADMA-induced dysregulation of respiration capacity and decreased mitochondrial ROS. Furthermore, in human hiPSCderived cardiomyocytes with impaired contractility under hypoxia and high ADMA, $\mathrm{rDDAH}-1$ treatment improved recovery and beating frequency $(p<0.05)$. rDDAH-1 treatment selectively modified I/R-induced myocardial cytokine expression resulting in reduction in pro-inflammatory cytokine IL-17A $(p<0.001)$ and increased expression of anti-inflammatory cytokines IL-10 and IL-13 ( $p<0.01)$. Further in vitro studies showed that IL-17A was the predominant and common cytokine modulated by ADMA-DDAH pathway in heart, cardiomyocytes and endothelial cells. These studies show that lowering ADMA by pharmacological treatment with $r D D A H-1$ reduced I/R injury, improved cardiac function, and improved cardiomyocyte bioenergetics and beating 
activity. These effects may be attributable to ADMA lowering in cardiomyocytes and preservation of cardiomyocyte mitochondrial function.

Significance Statement: The pathological role of ADMA has been demonstrated by its association with cardiovascular disease and mortality. Currently, pharmacological drugs to specifically lower ADMA are not available. The present study provides the first evidence that lowering of ADMA by recombinant rDDAH-1 decreased ischemia/reperfusion injury, improved post-ischemic cardiac function, and improved cardiomyocyte bioenergetics and beating activity. Our studies suggest that lowering of ADMA by pharmacologic treatment offers opportunity to develop new therapies for the treatment of cardiovascular and renal disease.

\section{Introduction}

High level of asymmetric dimethyl arginine (ADMA) accumulated during the conditions of ischemia, hypoxia or oxidative stress, has emerged as a risk factor for cardiovascular disease. Patients with preexisting atherosclerotic disease, diabetes, hypertension, and heart failure exhibit high levels of plasma ADMA that is associated with disease severity and mortality (Cavusoglu et al., 2010; Dowsett et al., 2020; Gać et al., 2020; Xuan et al., 2016). Meta analysis of results from 10 studies with 2195 patients suggested a strong correlation between plasma ADMA levels and mortality of heart failure patients (Pan et al., 2020). In addition, patients with chronic kidney disease show increasing plasma ADMA levels with the progression of kidney disease (Reddy et al., 
2015). Thus, ADMA may be a biomarker as well as a risk factor for cardiovascular disease.

ADMA is a competitive inhibitor of nitric oxide synthase (NOS) and arginine transport (Leiper and Nandi, 2011). A large number of early studies have focused on the effect of ADMA on vascular endothelium and vasoconstriction. Further studies in cellular systems and preclinical animal models have shown that high levels of ADMA may contribute to diverse pathological responses (Druhan et al., 2008; Mihout et al., 2011). In particular, ADMA induces the uncoupling of eNOS and generation of reactive oxygen species (ROS) (Druhan et al., 2008). These pathophysiological activities of ADMA may have considerable effects on vascular as well as cardiac function such as during heart attack, progression of chronic vascular disease and heart failure. Thus, it has been recognized that lowering of pathological ADMA may be a novel therapeutic target to reduce the risk of cardiovascular and kidney disease (Leiper and Nandi, 2011; Schwedhelm and Boger, 2011).

The major pathway modulating ADMA levels is through its metabolism by the enzyme dimethylarginine dimethylaminohydrolase (DDAH) (Leiper and Nandi, 2011; Schwedhelm and Boger, 2011). Two isoforms, DDAH-1 and DDAH-2, encoded by separate genes have been identified in mammalian species (Leiper and Nandi, 2011). Both enzymes catalyze the degradation of ADMA to citrulline and dimethylamine (Teerlink, 2005). Reduced expression or activity of DDAH has been found in animal models of cardiovascular and renal ischemia (Nakayama et al., 2014; Stuhlinger et al., 2007). More recently, it was discovered that the expression of DDAH-2 was reduced in human diabetic kidney (Barwinska et al., 2021). The role of DDAH as a modulator of 
ADMA in physiological and pathological states has been confirmed using rodent genetic models. Deletion of the DDAH-1 gene leads to increased ADMA levels and vascular dysfunction (Leiper et al., 2007). To the contrary, overexpression of DDAH-1 in transgenic animals or by adenoviral gene transfer leads to reduction in ADMA which is associated with improved vascular responses and reduced disease severity (Jacobi et al., 2010; Nakayama et al., 2014; Stuhlinger et al., 2007; Xu et al., 2017). DDAH gene transfer studies have suggested that the reduction of ADMA by increasing DDAH represents a new therapeutic approach to treat cardiovascular and renal disease (Arrigoni et al., 2010; Leiper and Nandi, 2011; Xu et al., 2017). At the present time, however, pharmacological drugs to specifically lower ADMA are not available.

In the present study, we have used recombinant DDAH-1 (rDDAH-1), which specifically metabolizes ADMA, as a pharmacological agent to investigate its potential therapeutic actions in myocardial ischemia-reperfusion $(\mathrm{I} / \mathrm{R})$ injury. We show that postischemic infusion of rDDAH-1 in a mouse model protected the heart against I/R-induced myocardial damage. rDDAH-1 treatment also improved beating frequency and recovery of human induced pluripotent stem cell (hiPSC) derived cardiomyocytes (iCMs) from hypoxia-and ADMA-induced stress. Cell culture studies showed that rDDAH-1 improved ADMA-dysregulated cardiomyocyte bioenergetics and reduced ADMA-induced inflammatory cytokines and ROS production. These results suggest that a rDDAH-1 based biotherapeutics may represent a novel approach for the treatment of ischemic heart disease.

\section{Methods and Materials}


Generation and Characterization of rDDAH-1. A human DDAH-1 homologous gene from Pseudomonas Aeruginosa-DDAH was cloned and expressed in E. coli. Coding sequence of DNA was synthesized and ligated into a pE-SUMO vector. Plasmid containing DDAH genes were transformed into BL21 (DE3) E. coli cells (EMD Millipore, USA), plated on LB agar plate with $50 \mu \mathrm{g} / \mathrm{mL}$ kanamycin and grown overnight. For preparation of purified DDAH, $50 \mathrm{~mL}$ culture were inoculated in $1 \mathrm{~L} \mathrm{LB}$ with $50 \mu \mathrm{g} / \mathrm{mL}$ kanamycin and grown to $0.8 \mathrm{OD}$ at $600 \mathrm{~nm}$ and then induced with IPTG. After $16 \mathrm{hrs}$, cells were collected by centrifugation, resuspended and lysed by sonication. DDAH in the supernatant was then purified using a Ni-sepharose column. DDAH purity was determined using SDS gel electrophoresis. DDAH activity was determined by generation of L-citrulline from ADMA using a colorimetric assay.(Knipp and Vasak, 2000). The purified recombinant DDAH used in this study is designated as rDDAH-1.

ADMA Measurement. ADMA in perfusate was analyzed by modification of previously published HPLC based method (de Jong and Teerlink, 2006; Teerlink, 2007). Test samples were prepared by solid-phase extraction (SPE) using an Oasis MCX Cartridge (Waters, USA). Briefly, the column was conditioned with $30 \%$ ammonium hydroxide:water:methanol (10:40:50 by volume) followed by ultrapure water. Plasma was mixed, vortexed, and spinned with cold methanol for extraction, mixed with PBS and loaded on to the column. The column was rinsed with $0.1 \mathrm{M} \mathrm{HCl}$ and methanol two times. Analytes were eluted with elution buffer and dried under nitrogen. Samples were reconstituted in ultrapure water and derivatized with ortho-phthaldialdehyde (OPA) reagent (Sigma-Aldrich, USA) (4:1 by volume). After mixing, the analytes were heated 
at $30{ }^{\circ} \mathrm{C}$ for $1 \mathrm{~min}$ and injected into the HPLC system, equipped with a fluorescent detector RF-10AXL (ex:340, em:455) and Chromolith ${ }^{\circledR}$ performance RP-18e column (100 x $4.6 \mathrm{~mm})$ (EMD Millipore, USA). Mobile phase A consisted of $25 \mathrm{mM}$ potassium phosphate buffer ( $\mathrm{pH} 6.5$ ), and mobile phase B was methanol/THF ( $97 / 3$ by volume). Chromatographic separation was performed at room temperature at a flow rate of 2 $\mathrm{mL} / \mathrm{min}(10-20 \%$ solvent $\mathrm{A})$.

ADMA Measurement in Cardiomyocyte. Cardiomyocytes prepared from adult mouse hearts were plated in 12 well plates at a density of 28,000 cells/well. Groups with ADMA $(10 \mu \mathrm{M})$ were incubated for 30 min and washed afterward. rDDAH-1 $(10 \mu \mathrm{g} / \mathrm{mL})$ was then added and cells were incubated for $1 \mathrm{hr}$ at $37^{\circ} \mathrm{C}$. After that, medium was removed and cells were washed once in medium without ADMA. Collected cells were lysed using M-PER lysis buffer and mixed, vortexed, and spinned with cold methanol for extraction and proceeded for ADMA measurement using HPLC.

Animals. Male C57BL/6J mice were purchased from the Jackson Laboratories (Bar Harbor, USA) and acclimated in the LARC facility for at least 5 days with a standard diet. 11-18 week old mice were employed for experiments. The animal protocol was reviewed and approved by the Institutional Animal Care and Use Committee of Indiana University. All animals received humane care in compliance with the Guide for the Care and Use of Laboratory Animals (NIH Pub. No. 85-23, revised 1996). 
Molecular Pharmacology Fast Forward. Published on January 18, 2022 as DOI: 10.1124/molpharm.121.000394

This article has not been copyedited and formatted. The final version may differ from this version.

\section{Isolated Mouse Heart Ischemia/Reperfusion Injury (Langendorff Model). Mouse}

hearts were isolated and subjected to Langendorff ischemia/reperfusion $(\mathrm{I} / \mathrm{R})$ as we previously described (Wang et al., 2014; Wang et al., 2009). Briefly, mice were heparinized (100 IU i.p.) and anesthetized with isoflurane, and hearts were rapidly excised. The aorta of the isolated heart was canulated and the heart was then perfused with oxygenated perfusion buffer containing ADMA $(1 \mu \mathrm{M})$ in an isovolumetric Langendorff mode $(70 \mathrm{mmHg})$. Left ventricular developed pressure (LVDP) was continuously recorded using a PowerLab 8 preamplifier/digitizer (AD Instruments Inc., Milford, MA). The maximal positive and negative values of the first derivative of pressure $(+d P / d t$ and $-d P / d t)$ were calculated using Labchart software. The coronary flow rate was measured by assessing the volume of pulmonary artery effluent per minute. Isolated mouse hearts were subjected to the $\mathrm{I} / \mathrm{R}$ by at least 15 min equilibration with perfusion buffer followed by 30 min global ischemia $\left(37^{\circ} \mathrm{C}\right)$ and 40 min reperfusion, and were randomly allocated to different experimental groups. Vehicle or rDDAH-1 was perfused during post-ischemic treatment. A dose-response study $(0,0.03,0.1$, and 0.3 $\mu \mathrm{g} / \mathrm{mL}$ ) was performed to determine the optimum dose of $r D D A H-1$ for protecting l/Rinduced myocardial dysfunction. Since the studies on all rDDAH-1 doses are not feasible in a single day, the experimental protocol was designed such that untreated controls $(0.0 \mu \mathrm{g} / \mathrm{mL})$ were included with each dose tested. This has led to more data points in the $0 \mu \mathrm{g} / \mathrm{mL}$ group than other groups. Also, $0.1 \mu \mathrm{g} / \mathrm{mL}$ of $\mathrm{rDDAH}-1$ was the optimum concentration and we performed an additional study to confirm it along with a control group. 
Infarct Size Measurement. The left ventricle (LV) from one set of mouse hearts +/rDDAH-1 $(0.1 \mu \mathrm{g} / \mathrm{mL})$ after Langendorff I/R was transversely sectioned into $\sim 1 \mathrm{~mm}$-thick frozen slices using a mouse heart slicer. The heart slices were then stained with $1 \%$ 2,3,5-triphenyltetrazolium chloride (TTC). Images were taken and the infarct area were analyzed using Image $(\mathrm{NIH})$. The infarct size (percentage) was calculated as total infarct weight per total LV weight from all slices as we previously reported (Wang et al., 2019). Infarct size measurement was conducted by an independent researcher blinded to the sample ID and the experimental groups.

Adult Mouse Cardiomyocyte Isolation. Cardiomyocytes were isolated from adult male mouse hearts as we previously described (Wang et al., 2019). Briefly, after mice were injected with heparin (100 IU, i.p.) and euthanized, the hearts were removed rapidly. Hearts were retrogradely perfused and digested with collagenase II. The cells were sequentially restored in calcium-contained buffer $(100,250,500$, or $1000 \mu \mathrm{mol} / \mathrm{L} \mathrm{CaCl}$ ), adult mouse cardiomyocytes were seeded into laminin-precoated plates with cardiomyocyte plating medium (MEM with glutamine $+2.5 \%$ FBS, $10 \mathrm{mM}$ BDM, and $1 \%$ Pen/Strep) and used for experiments.

Measurement of Mitochondrial Superoxide Production. Cardiomyocytes were treated with various concentrations of ADMA in the absence or presence of rDDAH-1 $(20 \mu \mathrm{g} / \mathrm{mL})$ for $2 \mathrm{hrs}$. After that, cardiomyocytes were loaded with $5 \mu \mathrm{M}$ MitoSOX Red (Thermo Fisher Scientific, USA) and incubated for 20 min at $37^{\circ} \mathrm{C}$. Intracellular MitoSox Red selectively targets mitochondria and is oxidized by mitochondrial superoxide but 
not by other ROS. After 20 min incubation, cells were washed once. The live-cell images of cardiomyocytes were taken using an Axio Observer Z1 motorized microscope (Zeiss, Germany) with a 10X objective. Red fluorescence intensity in an individual cardiomyocyte was quantified using Image $(\mathrm{NIH})$ as we previously stated (Wang et al., 2019).

Mitochondrial Respiration Capacity by Seahorse XF Cell Mito Stress Test. Oxygen consumption rate $(\mathrm{OCR})$ measurements were performed using a Seahorse Bioscience XF-96 instrument (Seahorse Biosciences, USA). One day before the assay, the sensor cartridge was hydrated overnight using the calibration buffer supplied by the manufacturer. Isolated mouse cardiomyocytes were plated in laminin-precoated XF96 cell culture microplate at 2500 cells/well. The cells were cultured overnight. On the day of the experiment, the cells are treated with ADMA in the absence or presence of $\mathrm{rDDAH}-1(20 \mu \mathrm{g} / \mathrm{mL})$ in supplemented XF medium and incubated at $37^{\circ} \mathrm{C}$ without $\mathrm{CO}_{2}$ for $2 \mathrm{hrs}$. The bioenergetics profile of cardiomyocytes was measured sequentially as baseline OCR, ATP-linked production by injection of $1 \mu \mathrm{M}$ oligomyocin, maximal uncoupled respiration by adding $0.75 \mu \mathrm{M}$ FCCP, and non-mitochondrial respiration by injection of $0.5 \mu \mathrm{M}$ rotenone and antimycin A.

hiPSC and Human Cardiomyocyte Culture. DiPS 1016 SevA hiPSCs (Harvard Stem Cell Institute, USA) derived from human skin fibroblasts (Passage number 40-50) were seeded and kept in culture on 1\% Geltrex (Invitrogen, USA)-coated culture flasks using mTeSR (StemCell Technologies, Canada) supplemented with 1\% penicillin (VWR, 
USA). At $80 \%$ confluency, hiPSCs were detached using Accutase (StemCell Technologies, Canada), and seeded into culture well plates in mTeSR1 media supplemented with $5 \mu \mathrm{M}$ Rho-associated, coiled-coil containing protein kinase (ROCK) inhibitor (StemCell Technologies, Canada). Cells were maintained with daily mTeSR1 media changes until 95\% confluency was reached. iCM differentiation from hiPSCs was adapted from a previously established protocol (Zhao et al., 2019). Briefly, at 95\% confluency, hiPSCs were treated with RPMI Medium 1640 (Life Technologies, USA) supplemented with 2\% B27 without insulin (Invitrogen, USA), $0.1 \mathrm{mM}$ betamercaptoethanol (Promega, USA), and 1\% penicillin (CM (-)) with the addition of $10 \mu \mathrm{M}$ Wht activator, CHIR99021 (CHIR) (Stemgent, USA) for exactly 24 hrs. Afterwards, media was replaced with CM (-) supplemented with $2 \mu \mathrm{M}$ CHIR on days 2 and 3 . On day 4, iCMs were treated with $\mathrm{CM}(-)$ media supplemented with the $5 \mu \mathrm{M}$ Wnt inhibitor, IWP-4 (Stemgent, USA). On day 6, media was replaced with CM (-). On day 9 of the differentiation, media was replaced with RPMI Medium 1640 supplemented with $2 \%$ B27 (Invitrogen, Carlsbad, CA, USA), $0.1 \mathrm{mM}$ beta-mercaptoethanol, and 1\% penicillin $(\mathrm{CM}(+))$. After day 9, media was changed every three days, and beating was observed generally by day 21 of differentiation.

In Vitro iCM Functional Analysis. After physiological beating of iCMs was observed, the baseline beat rate was recorded via brightfield videos using an Axio Observer Z1 motorized microscope and ORCA flash 4.0 camera (Hamamatsu, USA). Culture media was removed and replaced with RPMI Medium 1640 supplemented with B27 without antioxidants (Invitrogen, Carlsbad, CA), $0.1 \mathrm{mM}$ beta-mercaptoethanol, and 1\% 
penicillin that was pre-gassed with nitrogen to remove dissolved oxygen and the iCMs were incubated in anoxic conditions $\left(37^{\circ} \mathrm{C}, 94.9 \% \mathrm{~N}_{2}, 5 \% \mathrm{CO}_{2}\right.$, and $\left.0.1 \% \mathrm{O}_{2}\right)$ until beating ceased (typically between 4-8 hrs). Following anoxia, media was replaced with RPMI Medium 1640 supplemented with $30 \mu \mathrm{M}$ ADMA +/- $20 \mu \mathrm{g} / \mathrm{mL}$ rDDAH-1 and cultured in standard cell culture conditions for 72 hrs with timelapse videos taken 24-, and 72-hrs following anoxia treatment. A previously developed block-matching algorithm (Acun et al., 2019; Ellis et al., 2017) was applied to analyze the beating velocity of iCMs. This was done for all frames of the time-lapse video to create a time series of iCM beating velocity vectors. The peak velocity for each vector determined over time was averaged with that of all other vectors within each beating cluster to yield a single value representing the beating velocity of the entire field of view.

Cytokine Changes in Mouse Heart Perfusate. The cytokine content was determined in the perfusate from control and I/R mouse heart after Langendorff experiment, using Quantibody Mouse Cytokine Array 1 according to the manufacturer's instructions (RayBiotech, USA).

Cytokine Changes in Cell Culture. Mouse cardiomyocytes: Mouse cardiomyocytes used for investigation of cytokine changes were isolated as described in methods under Adult Mouse Cardiomyocyte Isolation. In order to mimic the ischemia-reperfusion of the heart, cardiomyocytes were subjected to hypoxia ( $5 \%$ oxygen) for 30 min then normoxia for $1 \mathrm{hr}$ in the presence or absence of $10 \mu \mathrm{M}$ ADMA or $10 \mu \mathrm{M}$ ADMA + 20 $\mu \mathrm{g} / \mathrm{mL} \mathrm{rDDAH}-1$. Supernatants were collected for cytokine assay. Human iCMs: 
Supernatants from human iCMs were collected for cytokine analysis at the end of 72 hours from the experiment described under In Vitro iCM Functional Analysis. HUVECs: Human umbilical vein endothelial cells (HUVECs) $(1,000,000$ cells/well) plated in six-well plates with serum-free defined medium. After overnight plating, cells were subjected to hypoxia (5\% oxygen) for 30 min then normoxia for $3 \mathrm{hrs}$ in the presence or absence of $10 \mu \mathrm{M}$ ADMA or $10 \mu \mathrm{M}$ ADMA+ $20 \mu \mathrm{g} / \mathrm{mL}$ rDDAH-1.

Supernatants were collected for cytokine assay. A custom designed cytokine kit from RayBiotech was used for cytokine determination in supernatant of human iCM and HUVEC. Slides were read in a GenePix scanner and performed analysis using QAnalyzer application.

NADPH Oxidase Assay. HUVECs were subjected to $5 \%$ hypoxia for $30 \mathrm{~min}$ and normoxia for $3 \mathrm{hrs}$ in the absence or presence of $10 \mu \mathrm{M}$ ADMA, $20 \mu \mathrm{g} / \mathrm{mL}$ rDDAH-1, or $10 \mu \mathrm{M}$ ADMA $+20 \mu \mathrm{g} / \mathrm{mL} \mathrm{rDDAH}-1$. Cells were then suspended in $100 \mu \mathrm{L}$ of KrebsHEPES buffer containing $0.5 \mathrm{mM}$ lucigenin. $50 \mu \mathrm{L}$ of suspension was transferred to assay plate and $5 \mu \mathrm{L}$ of $1 \mathrm{mM}$ NADPH was added. Photo emission expressed as relative light units (RLU) was measured every $1 \mathrm{~min}$ for $10 \mathrm{~min}$. The readings of the luminescence increased linearly within $5 \mathrm{~min}$, and the slope for the trend line was defined as the relative NOX activity, expressed as luminescence unit (RLU)/minute.

Statistical Analysis. The reported results were means \pm SD. Data was checked for distributions using Shapiro-Wilk normality test. Statistical significance was evaluated by unpaired t test when only two samples being compared, one-way ANOVA with 
Dunnett's post hoc testing (single experimental variable), or two-way ANOVA with

Sidak's comparison test (two experimental variables). Data sets not passed for normality test were evaluated using either Kruskal-Wallis test or Mann Whitney test. $P<$ 0.05 was considered statistically significant. ${ }^{*} P<0.05,{ }^{* \star} P<0.01,{ }^{* * \star} P<0.001,{ }^{* * \star} P<$ 0.0001. All statistical analyses were performed using the GraphPad Prism software.

\section{Results}

rDDAH-1 Lowered Extracellular and Intracellular ADMA in Cardiomyocytes. The role of DDAH in the metabolism of ADMA and cardiovascular pathophysiology has been demonstrated using rodent models by DDAH gene deletion or DDAH overexpression. However, pharmacological agents to specifically modulate ADMA levels in cellular systems in vitro and in disease models are not available. We have recently generated rDDAH-1 by cloning and expressing in E.coli. rDDAH-1 was highly effective in lowering ADMA in plasma in vitro and when administered to rats (Lee et al., 2021). Further, treatment with $\mathrm{rDDAH}-1$ protected kidney from $\mathrm{l} / \mathrm{R}$ injury in a rat model. Thus, $\mathrm{rDDAH}-1$ represents an important pharmacological agent for specific modulation of ADMA. In order to investigate ADMA lowering in heart, and its potential direct effect on cardiomyocytes, we first determined if rDDAH-1 can lower ADMA levels in the extracellular as well as intracellular milieu of cardiomyocytes. Figure 1 shows that addition of rDDAH-1 to the perfusion buffer used for the study of mouse heart I/R resulted in dose-dependent lowering of ADMA. Reduction in ADMA was achieved at concentrations as low as $0.1 \mu \mathrm{g} / \mathrm{mL}$ of rDDAH-1 $(0.88,95 \%$ confidence interval [Cl], $0.87-0.95$ vs. $0.96,95 \% \mathrm{Cl}, 0.91-1.01$ in group $0 ; \mathrm{p}<0.05)$ (Figure $1 \mathrm{~A})$. Figure $1 \mathrm{~B}$ 
shows that when added to the primary cultures of mouse cardiomyocyte, rDDAH-1 (10 $\mu \mathrm{g} / \mathrm{mL}$ ) produced about $50 \%$ lowering the intracellular ADMA within one hour. These data show that rDDAH-1 can directly modulate ADMA concentration within cardiomyocytes, providing the basis for investigating its effect on heart and cardiomyocyte function.

\section{rDDAH-1 Improved Cardiac Function and Reduced Myocardial Infarction}

Following I/R Injury. To determine the effect of rDDAH-1 on myocardial function, we performed a concentration-responsive study on isolated mouse hearts using an ex-vivo I/R model. In order to mimic the ADMA levels found in disease situation, especially following myocardial ischemic injury, $1 \mu \mathrm{M}$ of ADMA was added to the perfusion buffer. Mouse hearts were subjected to $30 \mathrm{~min}$ of ischemia and then $40 \mathrm{~min}$ of reperfusion. rDDAH-1 was infused into the isolated mouse hearts post ischemia. Figure 2 shows that addition of $\mathrm{rDDAH}-1$ to the perfusion buffer protected myocardium against $\mathrm{I} / \mathrm{R}$, as demonstrated by increase in cardiac contractility (LVDP: $44.08,95 \% \mathrm{Cl}, 32.92-55.25$ vs. $17.56,95 \% \mathrm{Cl}, 10.91-24.22$ in group $0, \mathrm{p}<0.01$; and $\mathrm{dP} / \mathrm{dt}$ : $54.21,95 \% \mathrm{Cl}, 34.86-$ 73.56 vs. $20.27,95 \% \mathrm{Cl}, 12.48-28.07, \mathrm{p}<0.01$ ) (Figure $2 \mathrm{~A}, 2 \mathrm{~B}$ ) and improved LV relaxation (-dP/dt) $(56.71,95 \% \mathrm{Cl}, 33.67-79.76$ vs. $22.86,95 \% \mathrm{Cl}, 13.5-32.32$, $\mathrm{p}<0.01$ ) (Figure $2 \mathrm{C})$. Cardiac rate pressure production (RPP $=$ LVDP $x$ heart rate) was increased by $\mathrm{rDDAH}-1$ treatment $(36.18,95 \% \mathrm{Cl}, 22.54-49.82$ in group 0.1 vs. 13.32 , $95 \% \mathrm{Cl}, 5.74-20.91$ in group $0, \mathrm{p}<0.01$ ) (Figure $2 \mathrm{D}$ ). $0.1 \mu \mathrm{g} / \mathrm{mL}$ of rDDAH-1 improved cardiac function of LVDP, + /- dP/dt and RPP by $1.5-1.7$ folds compared to group 0 . rDDAH-1 treatment did not change the heart rate (Figure 2E). An increased flow rate of 
coronary effluent (about $45 \%$ increase in group 0.3 [95.51, 95\% Cl, $75.35-115.7]$ vs. group 0 [65.88, 95\% $\mathrm{Cl}, 54.03-77.72]$ ) was observed in rDDAH-1 infused hearts (Figure $2 \mathrm{~F}$ ) which may result from vasodilation or myocardial protection. rDDAH-1 alone did not affect cardiac function or flow rate of coronary effluent in mouse hearts without I/R injury (Supplemental Figure 1). Post-ischemic usage of rDDAH-1 improved myocardial functional recovery, as demonstrated by about 150\% increase of LVDP compared with vehicle group after $\mathrm{I} / \mathrm{R}(\mathrm{p}<0.0001)$ (Figure $3 \mathrm{~A})$. In addition, the recovery of cardiac function was earlier than the vehicle control group. Histological analysis of heart also showed about 30\% reduction in myocardial infarct size in rDDAH-1 treated group $(15.9,95 \% \mathrm{Cl}, 12.65-19.15)$ as compared to the vehicle group $(22.24,95 \% \mathrm{Cl}$, $18.7-25.77$ ) (Figure 3B and 3C). Collectively, these results showed that pharmacological treatment with $\mathrm{rDDAH}-1$ protected myocardium from I/R injury and markedly improved recovery of LV function post ischemia.

\section{rDDAH-1 Improved Beating Frequency in Human Cardiomyocyte Culture. To}

further examine the direct effect of ADMA lowering on cardiomyocytes, we used induced pluripotent stem cell (iPSC) derived cardiomyocytes (iCM). Our group has previously characterized human iCMs and their beating response in vitro in both $2 \mathrm{D}$ and 3D cardiac models (Basara et al., 2021; Ellis et al., 2021; Ozcebe et al., 2021). iCMs were subjected to hypoxia and ADMA to mimic the condition that may occur during myocardial infarction. Addition of ADMA to iCM cultures immediately following anoxia inhibited beating frequency with about $25 \%$ reduction $(5.39,95 \% \mathrm{Cl}, 3.43-7.35$ vs. 7.4 , $95 \% \mathrm{Cl}, 5.21-9.59$ in control at 24 hours; $5.92,95 \% \mathrm{Cl}, 4.2-7.65$ vs. $7.5,95 \% \mathrm{Cl}$, 
$6.18-8.81$ in control at 72 hours after anoxia) $(p<0.05)$ and velocity recovery with about $30 \%$ decrease $(54.76,95 \%, 41.21-68.31$ vs. $77.22,95 \% \mathrm{Cl}, 62.81-91.63$ in control, $\mathrm{p}<0.01$ ) (Figure $4 \mathrm{~A}$ and $4 \mathrm{~B}$ ). We observed that treatment of iCMs with rDDAH-1 immediately following anoxia significantly reduced the detrimental effects of ADMA on beating rate $(7.49,95 \% \mathrm{Cl}, 6.18-8.81, \mathrm{p}<0.05)$ and velocity recovery $(76.25,95 \% \mathrm{Cl}$, $62.02-90.48, p<0.01$ ) within 72 hours. These data suggest that $\mathrm{rDDAH}-1$ provides a protective effect on human cardiomyocytes under conditions of high ADMA following anoxic conditions.

rDDAH-1 Improved Cardiomyocyte Mitochondrial Bioenergetics. Normal cellular metabolism is a prerequisite for maintaining cardiomyocyte function and survival. Impaired mitochondrial activity alters cellular metabolism and energy production. Previous studies on the role of ADMA in cardiovascular disease have focused on its inhibition of eNOS resulting in vascular dysfunction and vasoconstriction. The potential direct effect of ADMA on cardiomyocyte has not been well studied. In this study, we investigated the effect of ADMA and rDDAH-1 on the bioenergetic profile of mouse cardiomyocyte mitochondria using the Seahorse Cell Mito Stress Test assay (Figure 5A and 5B). Treatment of cardiomyocytes with ADMA increased OCR at the basal (205.1, $95 \% \mathrm{Cl}, 135.4-274.8$ vs. $135.1,95 \% \mathrm{Cl}, 82.76-187.4$ in control, $\mathrm{p}<0.05)$ and nonmitochondrial respiration level $(93.64,95 \% \mathrm{Cl}, 67.02-120.3$ vs. $57.84,95 \% \mathrm{CI}, 33.34-$ 82.34 in control, $p<0.05$ ) (Figure $5 \mathrm{C}$ and $5 \mathrm{D}$ ) while the maximal OCR was unchanged (Figure 5E). The maximum, uncontrolled OCR was obtained after addition of FCCP, which leads to a rapid consumption of oxygen without ATP generation. Notably, ADMA 
reduced spare respiratory capacity/reserve capacity (the ability of cells to respond to increased energy demand under stress) of cardiomyocytes (155.8, 95\% Cl, 103.2 208.4 vs. $215,95 \% \mathrm{Cl}, 170.5-259.6$ in control, $\mathrm{p}<0.05)$ (Figure 5F). These data suggest that ADMA directly alters mitochondrial respiratory function which could result in decreased tolerance to stress as under the conditions of hypoxia or ischemia. Importantly, treatment with rDDAH-1 reversed the ADMA-induced mitochondrial respiratory dysfunction in cardiomyocytes, including reduced basal $(125.2,95 \% \mathrm{Cl}$, 88.87 - 161.5, $\mathrm{p}<0.05)$ (Figure $5 \mathrm{C}$ ) and non-mitochondrial respiration $(43.97,95 \% \mathrm{Cl}$, 32.92 - 55.02, $\mathrm{p}<0.01$ ) (Figure 5D), as well as improved spare respiratory capacity $(254.8,95 \% \mathrm{Cl}, 210-299.7, \mathrm{p}<0.01)$ (Figure 5F). In normal adult cardiomyocytes, treatment with rDDAH-1 alone did not impact mitochondrial energetics in (Supplemental Figure 2). These results demonstrate that altered cardiomyocyte respiration under pathological levels of ADMA can be reversed by treatment with rDDAH-1.

rDDAH-1 Reversed the Effect of ADMA on Mitochondrial ROS Production. It is known that oxidative stress, particularly mitochondrial-generated ROS, can substantially contribute to cardiac dysfunction. Therefore, we further investigated if ADMA and rDDAH-1 directly affected the generation of ROS in mitochondria. Mitochondrial ROS production was determined in isolated mouse cardiomyocytes in the presence or absence of ADMA and rDDAH-1 using the MitoSoX Red probe. In this assay, superoxide production in mitochondria is detected by the increase in fluorescence intensity of MitoSoX Red (Figure 6A). Addition of ADMA (3, 10, and $30 \mu \mathrm{M})$ to cardiomyocytes resulted in the elevation of oxygen free radical production within 1 hour 
$(1.79,95 \% \mathrm{Cl}, 1.65-1.92 ; 1.7,95 \% \mathrm{Cl}, 1.53-1.86$, and $2.18,95 \% \mathrm{Cl}, 1.94-2.41$ in

groups of 3,10 , and $30 \mu \mathrm{M}$ of ADMA vs. $0.998,95 \% \mathrm{Cl}, 0.9-1.1$ in group $0, p<0.0001$ )

(Figure 6B). Treatment with rDDAH-1 decreased ADMA-induced mitochondrial

superoxide production $(1.31,95 \% \mathrm{Cl}, 1.14-1.48$ and $1.15,95 \% \mathrm{Cl}, 0.98-1.32$ in 3

and $10 \mu \mathrm{M}$ ADMA + rDDAH-1, respectively, $\mathrm{p}<0.0001$ ) (Figure 6C). ADMA and rDDAH-

1 treatment did not change NADPH oxidase activity (Supplement Figure 3), suggesting

that the induction of ROS by ADMA may be largely due to its previously well

documented effect on eNOS uncoupling (Badran et al., 2016; Druhan et al., 2008;

Janaszak-Jasiecka et al., 2021; Karbach et al., 2014; Sud et al., 2008). These data

show that rDDAH-1 can reduce ROS generation under the condition of high ADMA as observed following ischemia or in chronic heart failure.

\section{rDDAH-1 Modified Inflammatory Cytokine Expression in Mouse Heart Following}

I/R and Cardiac Cells in Vitro. Cardiac I/R is associated with increased inflammatory cytokine expression which can produce further injury to the heart. Previous studies have not examined the cytokine profile when the heart is subjected to high ADMA and I/R.

We examined a large panel of cytokines released in the coronary effluent obtained from the isolated mouse hearts subjected to $\mathrm{I} / \mathrm{R}$ with or without $\mathrm{rDDAH}-1$ treatment. Our results show that in mouse $\mathrm{I} / \mathrm{R}$ heart, $\mathrm{rDDAH}-1$ selectively reduced proinflammatory cytokine IL-17A (327.9, 95\% Cl, $91.02-564.9$ vs. $1796,95 \% \mathrm{Cl}, 1021-2571$ in vehicle, $\mathrm{p}<0.001)$ and increased anti-inflammatory cytokine $\mathrm{IL}-10(75764,95 \% \mathrm{Cl}, 59874-$ 91654 vs. $33437,95 \% \mathrm{Cl}, 11687-55186$ in vehicle, $\mathrm{p}<0.01)$ and IL-13 $(7641,95 \% \mathrm{Cl}$, $5451-9831$ vs. $2620,95 \% \mathrm{Cl}, 659.7-4580$ in vehicle, $\mathrm{p}<0.01$ ) (Figure 7B). 
Further investigation of the effect of ADMA and rDDAH-1 on cytokine expression in mouse cardiomyocytes, human iCM and endothelial cells showed that changes in IL17A in response to ADMA or ADMA+rDDAH-1 was common to these cell type (Figure 8). It is expected that the cytokine expression profile of the different cells subjected to ischemia-reperfusion may be different. This may be due to difference in species (mouse vs human cells), cell type and the cell culture conditions required for maintenance of different cell type (Figure 8A). Despite the differences, the effect of ADMA and rDDAH-1 was limited to specific cytokines. We observed that ADMA increased IL-17A production in human iCM $(64,95 \% \mathrm{Cl}, 31.71-96.29$ vs. $27.67,95 \% \mathrm{Cl}, 6.98-48.35$ in control, $\mathrm{p}<0.01$ ) (Figure $8 \mathrm{C}$ ), mouse cardiomyocytes $(48.1,95 \% \mathrm{Cl}, 18.32-77.88$ vs. $22.67,95 \%$ Cl, $4.36-40.98$ in control, $p<0.05)$ (Figure 8D), and HUVEC (141, 95\% Cl, $76.56-$ 205.4 vs. $59,95 \% \mathrm{Cl}, 39.13-78.87$ in control, p<0.01) (Figure $8 \mathrm{E}$ ). Importantly, rDDAH-1 decreased ADMA-induced IL-17A levels in human iCM $(39,95 \% \mathrm{Cl}, 23.89$ $54.11, \mathrm{p}<0.05)$ and mouse cardiomyocytes $(25.63,95 \% \mathrm{Cl}, 17.31-33.96, \mathrm{p}<0.05)$. Our studies, for the first time, reveal that the effect of ADMA and rDDAH-1 on IL-17A was common to all cells. These results suggest that increased IL-17A may be an important mechanism of mediating the inflammatory response to ADMA.

\section{Discussion}

Patients with cardiovascular disease risk factors including hypertension, hypercholesterolemia diabetes, and heart failure exhibit high blood levels of ADMA. High ADMA levels are associated with cardiovascular disease progression and mortality (Cavusoglu et al., 2010; Dowsett et al., 2020; Gać et al., 2020; Xuan et al., 2016). The 
findings from clinical studies are further substantiated by the induction or exacerbation of disease in animal models in which ADMA levels are increased either by DDAH gene deletion (Leiper et al., 2007) or by ADMA administration (Achan et al., 2003). Based on the clinical and preclinical studies, ADMA is considered as a risk factor for vascular dysfunction and cardiovascular disease.

In the heart, high levels of ADMA may reduce NO bioavailability inducing endothelial dysfunction and vasoconstriction. In addition, increasing evidence suggests that the pathological action of ADMA may extend beyond its vasoconstriction activity. ADMA has been found to affect mitochondrial function in type 2 diabetic rats by inducing oxygen free radicals which may contribute to its direct effect on myocardial cell metabolism (Xiong et al., 2020). Recent studies have shown that treatment with ADMA directly inhibited the maximum contraction tension of papillary muscle (Xiong et al., 2021). Moreover, cardiac-specific deletion of DDAH-1 gene produced larger infarct size and impaired LV function in a mouse model of myocardial infarction (Hou et al., 2018). Thus, extravascular effect of ADMA, particularly in the heart, may be an important mechanism for its pathological actions in ischemic heart disease and heart failure. In this study, we have for the first time used a pharmacological approach to specifically lower ADMA in the heart and investigated its effect on the isolated cardiomyocytes. We show that the intracellular levels of ADMA in cardiomyocytes can be directly modulated by exogenously added rDDAH-1. We show that direct treatment with ADMA metabolizing enzyme DDAH reduced myocardial ischemic injury and preserved cardiac function in mouse model of global ischemia. Treatment with rDDAH-1 also decreased myocardial infarct size after I/R. Notably, rDDAH-1 treatment reduced inflammatory 
cytokine IL-17A while increased anti-inflammatory cytokines IL-10 and IL-13. These findings suggest the ADMA lowering may prevent I/R-induced inflammatory response which may cause further myocardial damage. Further in vitro investigation of the effect of ADMA and rDDAH-1 on cardiomyocytes and endothelial cells showed that IL-17A expression was uniformly modulated by ADMA in these cells. In this regard, it is important to note that previous studies have shown that IL-17A plays an important role in ischemia reperfusion injury and cardiac remodeling. In patients with myocardial infarction high serum levels of IL-17 have been observed (Garza-Reyes et al., 2020; Jafarzadeh et al., 2009). Also, genetic association IL-17 with carotid medial-intimal thickening (Wu et al., 2018) and coronary artery disease (Ghaznavi and Soltanpour, 2020) has been reported. These clinical and preclinical studies have suggested that IL17 is an important player in the post-infarct inflammation and ventricular remodeling in preclinical model of ischemia (Chang et al., 2018; Liao et al., 2012; Zhou et al., 2014). Our studies are the first to reveal a link between IL-17A and ADMA suggesting that IL17A may play an important role in ADMA mediated inflammation and cardiovascular disease.

Our studies show that direct treatment with rDDAH-1 improved cardiomyocyte mitochondrial respiratory dysfunction. rDDAH-1 treatment improved the ADMA induced basal and non-mitochondrial respiration and impaired spare respiratory capacity. Since cardiomyocytes undergo major metabolic and biochemical shifts during myocardial $\mathrm{l} / \mathrm{R}$, the deprivation of oxygen and nutrients alters mitochondrial function and ATP production (Garbern and Lee, 2021; McDougal and Dewey, 2017). The improvement in mitochondrial respiratory capacity observed in our studies suggest that rDDAH-1 may 
increase the ability of myocardium to respond to the increased energy demand under stress condition such as ischemia and oxidative stress. Our findings suggest that in addition to its effect on vascular function, high levels of ADMA may directly induce cardiac dysfunction which can be prevented by treatment with rDDAH-1.

We further show that addition of rDDAH-1 reduced the ADMA induced ROS in cardiomyocyte mitochondria. The role of ROS in mitochondrial damage and bioenergetics has been well documented. ROS can induce opening of the mitochondrial permeability transition pore (mPTP) and destroy the intracellular redox balance leading to oxidative stress, causing extensive damage to protein. Previous studies have shown that ADMA induces the generation of ROS by uncoupling of NOS (Druhan et al., 2008). Our studies show that under the condition of high ADMA, therapeutic treatment with rDDAH-1 reduced mitochondrial ROS generation. Thus, pharmacological lowering of ADMA may reduce oxidative stress and prevent ROS mediated mitochondrial dysfunction which has been shown to contribute to ischemic heart disease and progression of heart failure.

Our studies with hiPSC derived human cardiomyocytes have provided further evidence for a direct effect of ADMA lowering on cardiomyocyte function. iCMs have been previously characterized for their cardiomyocyte like-properties and beating behavior. Transient exposure to ADMA dramatically reduced beating frequency and velocity during iCM recovery from hypoxia induced beating cessation. Treatment with rDDAH-1 $(\mathrm{p}<0.05)$ improved the beating rate and the recovery from hypoxia and ADMA induced stress. These studies further support a direct effect of ADMA on cardiomyocyte and functional improvement by treatment with rDDAH-1. 
A limitation of our studies is the use of recombinant PA-DDAH (rDDAH-1) for the investigation of the effect of pharmacological lowering of ADMA in vitro and in vivo. We have cloned and expressed both human DDAH-1 and PA-DDAH in E. Coli. We found that as compared to the human DDAH-1, the PA-DDAH is expressed at much higher levels and was 10-30 fold more active. More importantly, PA-DDAH was stable under the experimental conditions required to perform the current studies. With the proof of efficacy with PA-DDAH, further studies will be undertaken on the expression of recombinant human DDAH-1, its protein biochemistry and stability for future pharmacological studies.

In summary, our studies have shown that pharmacological modulation of cellular ADMA by $\mathrm{rDDAH}-1$ can provide an important approach to investigate the pathological effects of ADMA in various disease states. High levels of ADMA are considered a risk factor for cardiovascular disease. Therefore, the reduction of ADMA by a pharmacological agent represents an important strategy for the development of new drugs for the treatment of cardiovascular disease.

\section{Acknowledgements}

The authors thank Dr. Chandan K. Sen at IU School of Medicine (IUSM) for allowing access to the Seahorse Bioscience XF-96 instrument and thank Dr. Kanhaiya Singh from Division of Plastic Surgery at IUSM for his assistance in acquiring the Seahorse data.

\section{Contributions}


Participated in research design: Lee, Singh, Zorlutuna, Wang

Conducted experiments: Lee, Scott, Ellis, Wang

Contributed new reagents or analytic tools: Lee, Wang

Performed data analysis: Lee, Scott, Ellis, Wang

Wrote or contributed to the writing of the manuscript: Lee, Singh, Wang

\section{The authors declare no conflicts of interest.}

\section{References}

Achan V, Broadhead M, Malaki M, Whitley G, Leiper J, MacAllister R and Vallance P (2003) Asymmetric dimethylarginine causes hypertension and cardiac dysfunction in humans and is actively metabolized by dimethylarginine dimethylaminohydrolase. Arterioscler Thromb Vasc Biol 23(8): 1455-1459.

Acun A, Nguyen TD and Zorlutuna P (2019) In vitro aged, hiPSC-origin engineered heart tissue models with age-dependent functional deterioration to study myocardial infarction. Acta Biomater 94: 372-391.

Arrigoni F, Ahmetaj B and Leiper J (2010) The biology and therapeutic potential of the DDAH/ADMA pathway. Curr Pharm Des 16(37): 4089-4102.

Badran M, Abuyassin B, Golbidi S, Ayas N and Laher I (2016) Uncoupling of Vascular Nitric Oxide Synthase Caused by Intermittent Hypoxia. Oxid Med Cell Longev 2016: 2354870.

Barwinska D, El-Achkar TM, Melo Ferreira R, Syed F, Cheng Y-H, Winfree S, Ferkowicz MJ, Hato T, Collins KS, Dunn KW, Kelly KJ, Sutton TA, Rovin BH, Parikh SV, 
Molecular Pharmacology Fast Forward. Published on January 18, 2022 as DOI: 10.1124/molpharm.121.000394

This article has not been copyedited and formatted. The final version may differ from this version.

Phillips CL, Dagher PC and Eadon MT (2021) Molecular characterization of the human kidney interstitium in health and disease. Science Advances 7(7): eabd3359.

Basara G, Ozcebe SG, Ellis BW and Zorlutuna P (2021) Tunable human myocardium derived decellularized extracellular matrix for 3D bioprinting and cardiac tissue engineering. bioRxiv: 2021.2003.2030.437600.

Cavusoglu E, Ruwende C, Chopra V, Poludasu S, Yanamadala S, Frishman WH, Eng C, Pinsky DJ and Marmur JD (2010) Relation of baseline plasma ADMA levels to cardiovascular morbidity and mortality at two years in men with diabetes mellitus referred for coronary angiography. Atherosclerosis 210(1): 226-231.

Chang SL, Hsiao YW, Tsai YN, Lin SF, Liu SH, Lin YJ, Lo LW, Chung FP, Chao TF, Hu YF, Tuan TC, Liao JN, Hsieh YC, Wu TJ, Higa S and Chen SA (2018) Interleukin-17 enhances cardiac ventricular remodeling via activating MAPK pathway in ischemic heart failure. $\mathrm{J} \mathrm{Mol} \mathrm{Cell} \mathrm{Cardiol} \mathrm{122:} \mathrm{69-79.}$

de Jong S and Teerlink T (2006) Analysis of asymmetric dimethylarginine in plasma by HPLC using a monolithic column. Analytical Biochemistry 353(2): 287-289.

Dowsett L, Higgins E, Alanazi S, Alshuwayer NA, Leiper FC and Leiper J (2020) ADMA:

A Key Player in the Relationship between Vascular Dysfunction and Inflammation in Atherosclerosis. J Clin Med 9(9).

Druhan LJ, Forbes SP, Pope AJ, Chen C-A, Zweier JL and Cardounel AJ (2008) Regulation of eNOS-Derived Superoxide by Endogenous Methylarginines. Biochemistry 47(27): 7256-7263. 
Ellis BW, Acun A, Can UI and Zorlutuna P (2017) Human iPSC-derived myocardium-onchip with capillary-like flow for personalized medicine. Biomicrofluidics 11(2): 024105.

Ellis BW, Traktuev DO, Merfeld-Clauss S, Can UI, Wang M, Bergeron R, Zorlutuna P and March KL (2021) Adipose stem cell secretome markedly improves rodent heart and human induced pluripotent stem cell-derived cardiomyocyte recovery from cardioplegic transport solution exposure. Stem Cells 39(2): 170-182.

Gać P, Poręba M, Jurdziak M, Trzmielewska E, Gocławska K, Derkacz A, Mazur G, Szuba A and Poręba R (2020) Cardiovascular risk factors and the concentration of asymmetric dimethylarginine. Adv Clin Exp Med 29(1): 63-70.

Garbern JC and Lee RT (2021) Mitochondria and metabolic transitions in cardiomyocytes: lessons from development for stem cell-derived cardiomyocytes. Stem Cell Research \& Therapy 12(1): 177.

Garza-Reyes MG, Mora-Ruíz MD, Chávez-Sánchez L, Madrid-Miller A, CabreraQuintero AJ, Maravillas-Montero JL, Zentella-Dehesa A, Moreno-Ruíz L, PastorSalgado S, Ramírez-Arias E, Pérez-Velázquez N, Chávez-Rueda AK, BlancoFavela F, Vazquez-Gonzalez WG and Contreras-Rodríguez A (2020) Effect of Interleukin-17 in the Activation of Monocyte Subsets in Patients with ST-Segment Elevation Myocardial Infarction. Journal of Immunology Research 2020: 5692829.

Ghaznavi H and Soltanpour MS (2020) Association study between rs2275913 genetic polymorphism and serum levels of IL-17A with risk of coronary artery disease. Mol Biol Res Commun 9(1): 35-40. 
Hou L, Guo J, Xu F, Weng X, Yue W and Ge J (2018) Cardiomyocyte dimethylarginine dimethylaminohydrolase 1 attenuates left-ventricular remodeling after acute myocardial infarction: involvement in oxidative stress and apoptosis. Basic Res Cardiol 113(4): 28.

Jacobi J, Maas R, Cardounel AJ, Arend M, Pope AJ, Cordasic N, Heusinger-Ribeiro J, Atzler D, Strobel J, Schwedhelm E, Böger RH and Hilgers KF (2010) Dimethylarginine dimethylaminohydrolase overexpression ameliorates atherosclerosis in apolipoprotein E-deficient mice by lowering asymmetric dimethylarginine. Am J Pathol 176(5): 2559-2570.

Jafarzadeh A, Esmaeeli-Nadimi A, Nough H, Nemati M and Rezayati MT (2009) Serum levels of interleukin (IL)-13, IL-17 and IL-18 in patients with ischemic heart disease. Anadolu Kardiyol Derg 9(2): 75-83.

Janaszak-Jasiecka A, Siekierzycka A, Płoska A, Dobrucki IT and Kalinowski L (2021) Endothelial Dysfunction Driven by Hypoxia-The Influence of Oxygen Deficiency on NO Bioavailability. Biomolecules 11(7).

Karbach S, Wenzel P, Waisman A, Munzel T and Daiber A (2014) eNOS uncoupling in cardiovascular diseases--the role of oxidative stress and inflammation. Curr Pharm Des 20(22): 3579-3594.

Knipp M and Vasak M (2000) A colorimetric 96-well microtiter plate assay for the determination of enzymatically formed citrulline. Anal Biochem 286(2): 257-264.

Lee Y, Mehrotra P, Basile D, Ullah M, Singh A, Skill N, Younes ST, Sasser J, Shekhar A and Singh J (2021) Specific Lowering of Asymmetric Dimethylarginine by Pharmacological Dimethylarginine Dimethylaminohydrolase Improves Endothelial 
Molecular Pharmacology Fast Forward. Published on January 18, 2022 as DOI: 10.1124/molpharm.121.000394

This article has not been copyedited and formatted. The final version may differ from this version.

Function, Reduces Blood Pressure and Ischemia-Reperfusion Injury. Journal of Pharmacology and Experimental Therapeutics 376(2): 181.

Leiper $\mathrm{J}$ and Nandi M (2011) The therapeutic potential of targeting endogenous inhibitors of nitric oxide synthesis. Nat Rev Drug Discov 10(4): 277-291.

Leiper J, Nandi M, Torondel B, Murray-Rust J, Malaki M, O'Hara B, Rossiter S, Anthony S, Madhani M, Selwood D, Smith C, Wojciak-Stothard B, Rudiger A, Stidwill R, McDonald NQ and Vallance P (2007) Disruption of methylarginine metabolism impairs vascular homeostasis. Nature Medicine 13(2): 198-203.

Liao YH, Xia N, Zhou SF, Tang TT, Yan XX, Lv BJ, Nie SF, Wang J, Iwakura Y, Xiao H, Yuan J, Jevallee H, Wei F, Shi GP and Cheng X (2012) Interleukin-17A contributes to myocardial ischemia/reperfusion injury by regulating cardiomyocyte apoptosis and neutrophil infiltration. J Am Coll Cardio/ 59(4): 420429.

McDougal AD and Dewey CF, Jr. (2017) Modeling oxygen requirements in ischemic cardiomyocytes. J Biol Chem 292(28): 11760-11776.

Mihout F, Shweke N, Bigé N, Jouanneau C, Dussaule J-C, Ronco P, Chatziantoniou C and Boffa J-J (2011) Asymmetric dimethylarginine (ADMA) induces chronic kidney disease through a mechanism involving collagen and TGF- $\beta 1$ synthesis. The Journal of Pathology 223(1): 37-45.

Nakayama Y, Ueda S, Yamagishi S, Obara N, Taguchi K, Ando R, Kaida Y, Iwatani R, Kaifu K, Yokoro M, Toyonaga M, Kusumoto T, Fukami K and Okuda S (2014) Asymmetric dimethylarginine accumulates in the kidney during ischemia/reperfusion injury. Kidney Int 85(3): 570-578. 
Ozcebe SG, Bahcecioglu G, Yue XS and Zorlutuna P (2021) Effect of cellular and ECM aging on human iPSC-derived cardiomyocyte performance, maturity and senescence. Biomaterials 268: 120554.

Pan W, Lian B, Lu H, Liao P, Guo L and Zhang M (2020) Prognostic Value of Asymmetric Dimethylarginine in Patients with Heart Failure: A Systematic Review and Meta-analysis. Biomed Res Int 2020: 6960107-6960107.

Reddy YS, Kiranmayi VS, Bitla AR, Krishna GS, Rao PVLNS and Sivakumar V (2015) Nitric oxide status in patients with chronic kidney disease. Indian J Nephrol 25(5): 287-291.

Schwedhelm E and Boger RH (2011) The role of asymmetric and symmetric dimethylarginines in renal disease. Nat Rev Nephrol 7(5): 275-285.

Stuhlinger MC, Conci E, Haubner BJ, Stocker EM, Schwaighofer J, Cooke JP, Tsao PS, Pachinger O and Metzler B (2007) Asymmetric Dimethyl L-Arginine (ADMA) is a critical regulator of myocardial reperfusion injury. Cardiovasc Res 75(2): 417-425.

Sud N, Wells SM, Sharma S, Wiseman DA, Wilham J and Black SM (2008) Asymmetric dimethylarginine inhibits HSP90 activity in pulmonary arterial endothelial cells: role of mitochondrial dysfunction. Am J Physiol Cell Physiol 294(6): C1407-1418.

Teerlink T (2005) ADMA metabolism and clearance. Vascular Medicine 10(2_suppl): S73-S81.

Teerlink T (2007) HPLC analysis of ADMA and other methylated I-arginine analogs in biological fluids. Journal of Chromatography B 851(1): 21-29. 
Wang L, Gu H, Turrentine M and Wang M (2014) Estradiol treatment promotes cardiac stem cell (CSC)-derived growth factors, thus improving CSC-mediated cardioprotection after acute ischemia/reperfusion. Surgery 156(2): 243-252.

Wang M, Smith K, Yu Q, Miller C, Singh K and Sen CK (2019) Mitochondrial connexin 43 in sex-dependent myocardial responses and estrogen-mediated cardiac protection following acute ischemia/reperfusion injury. Basic Res Cardio/ 115(1): 1.

Wang M, Wang Y, Weil B, Abarbanell A, Herrmann J, Tan J, Kelly M and Meldrum DR (2009) Estrogen receptor beta mediates increased activation of PI3K/Akt signaling and improved myocardial function in female hearts following acute ischemia. Am J Physiol Regul Integr Comp Physiol 296(4): R972-978.

Wu TW, Chou CL, Chen YC, Juang YL and Wang LY (2018) Associations of Common Genetic Variants on IL-17 Genes and Carotid Intima-Media Thickness. J Atheroscler Thromb 25(11): 1156-1167.

Xiong Y, Hai C-X, Fang W-J, Lei Y-P, Li X-M and Zhou X-K (2020) Endogenous asymmetric dimethylarginine accumulation contributes to the suppression of myocardial mitochondrial biogenesis in type 2 diabetic rats. Nutr Metab (Lond) 17: 72-72.

Xiong Y, He Y-L, Li X-M, Nie F and Zhou X-K (2021) Endogenous asymmetric dimethylarginine accumulation precipitates the cardiac and mitochondrial dysfunctions in type 1 diabetic rats. European Journal of Pharmacology 902: 174081. 
Xu X, Zhang P, Kwak D, Fassett J, Yue W, Atzler D, Hu X, Liu X, Wang H, Lu Z, Guo H, Schwedhelm E, Boger RH, Chen P and Chen Y (2017) Cardiomyocyte dimethylarginine dimethylaminohydrolase-1 (DDAH1) plays an important role in attenuating ventricular hypertrophy and dysfunction. Basic Res Cardio/ 112(5): 55.

Xuan C, Tian QW, Li H, Zhang BB, He GW and Lun LM (2016) Levels of asymmetric dimethylarginine (ADMA), an endogenous nitric oxide synthase inhibitor, and risk of coronary artery disease: A meta-analysis based on 4713 participants. Eur $\mathrm{J}$ Prev Cardiol 23(5): 502-510.

Zhao M, Tang Y, Zhou Y and Zhang J (2019) Deciphering Role of Wnt Signalling in Cardiac Mesoderm and Cardiomyocyte Differentiation from Human iPSCs: Fourdimensional control of Wnt pathway for hiPSC-CMs differentiation. Scientific Reports 9(1): 19389.

Zhou SF, Yuan J, Liao MY, Xia N, Tang TT, Li JJ, Jiao J, Dong WY, Nie SF, Zhu ZF, Zhang WC, Lv BJ, Xiao H, Wang Q, Tu X, Liao YH, Shi GP and Cheng X (2014) IL-17A promotes ventricular remodeling after myocardial infarction. $J$ Mol Med (Berl) 92(10): 1105-1116.

Financial Disclosure: No author has an actual or perceived conflict of interest with the contents of this article.

This work was supported by the Indiana Clinical and Translational Sciences Institute [Grant UL1TR001108] from the National Institutes of Health, National Center for 
Advancing Translational Sciences, Clinical, and Translational Sciences Award; AIM grant from IU Health and Indiana-CTSI. The content is solely the responsibility of the authors and does not necessarily represent the official views of the National Institutes of Health.

Reprint request to: Meijing Wang, MD

950 W. Walnut Street, R2 E319

Indianapolis, Indiana 46202

meiwang@iupui.edu

$317-274-0827$ 


\section{Figure Legends}

Figure 1. rDDAH-1 lowered ADMA in heart perfusate and cardiomyocytes in vitro.

A. Different concentrations of rDDAH-1 were added to the perfusate solution containing $1 \mu \mathrm{M}$ ADMA used for mouse heart perfusion in the ischemia-reperfusion studies and incubated at $37^{\circ} \mathrm{C}$ for $1 \mathrm{hr}$. ADMA remaining in the perfusate was then determined. The data show ${ }^{*} p<0.05,{ }^{* * *} p<0.001$ using One-way ANOVA with Dunnett's post hoc test. B. Reduction of intracellular ADMA in cardiomyocytes when incubated in the presence of rDDAH-1. Cardiomyocytes prepared from adult mouse hearts were treated with or without ADMA (10 $\mu \mathrm{M})$ for 30 min (3 wells/group). rDDAH-1 $(10 \mu \mathrm{g} / \mathrm{mL})$ was then added and cells were incubated for $1 \mathrm{hr}$ at $37^{\circ} \mathrm{C}$. Extracellular ADMA was removed by washing the cells with medium without ADMA. Intracellular ADMA remaining in cell extract was determined. The experiments were conducted in cardiomyocytes isolated from four mouse hearts. IC: The data show ${ }^{*} p<0.05,{ }^{* *} p<0.01$, unpaired t-test. All data are expressed as mean \pm SD.

Figure 2. rDDAH-1 improved cardiac function in the mouse $\mathbf{I} / \mathbf{R}$ model. Left ventricular (LV) function was determined in isolated mouse heart using the Langendorff preparation. Isolated mouse hearts were equilibrated $(\mathrm{Eq})$ with the perfusion buffer and the cardiac functions were determined (values at Eq) for each animal. Each mouse heart was then subjected to $30 \mathrm{~min}$ of warm ischemia followed by $60 \mathrm{~min}$ of reperfusion. Indicated concentrations of rDDAH-1 was infused during the reperfusion period and cardiac functions were determined. The data for the groups are expressed as $\%$ of cardiac function at Eq for each mouse heart. LV function was evaluated as: $\mathbf{A}$. LVDP = 
LV systolic pressure - LV diastolic pressure. B. $\mathrm{dP} / \mathrm{dt}$ (the maximum first derivative of LV pressure with respect to time). C. - $\mathrm{dP} / \mathrm{dt}$ (the maximum negative value of the first derivative of the pressure with respect to time). D. RPP (rate pressure product $=$ LVDP $X$ Heart rate). E. Heart rate at end of reperfusion among groups. $\mathbf{F}$. The flow rate for coronary effluent was measured at 20 min post ischemia. Each symbol indicates the individual value from an isolated mouse heart. All data are given as mean \pm SD. Significance determined by control-compared one-way ANOVA with Dunnett's post hoc test. ${ }^{*} \mathrm{p}<0.05,{ }^{* *} \mathrm{p}<0.01$.

Figure 3. rDDAH-1 improved myocardial functional recovery and reduced cardiac damage in mouse I/R injury model. A. shows change in LVDP over time in control and of rDDAH-1 $(0.1 \mu \mathrm{g} / \mathrm{mL})$ following 30 min warm ischemia and 40 min reperfusion $(\mathrm{I} / \mathrm{R})$. The data are ${ }^{* * \star *} \mathrm{p}<0.0001$ determined by two-way ANOVA with Sidak's comparison test. B. Representative photographs of transverse slices with TTC staining from mouse hearts without or with post-ischemic rDDAH-1 treatment following I/R. C. Myocardial Infarct size as determined by TTC staining of control and rDDAH-1 $(0.1 \mu \mathrm{g} / \mathrm{mL})$ treated hearts. The data show ${ }^{* *} \mathrm{p}<0.01$, unpaired t-test. All data are given as mean $\pm \mathrm{SD}$.

Figure 4. rDDAH-1 improved contractility recovery in ADMA treated iCMs following anoxia. iCM were subjected to hypoxia by incubation in $0.1 \%$ oxygen and deoxygenated media for $6 \mathrm{hrs}$ until beating ceased. The cells were then transferred to normoxia and media was replaced with oxygenated media in the presence or absence 
of ADMA (30 $\mu \mathrm{M})$ or rDDAH-1 $(20 \mu \mathrm{g} / \mathrm{mL})$. A. Changes in beating velocity was measured at baseline (0 hr), 24 and $72 \mathrm{hrs}$ after hypoxia. B. Beating rate recovery were determined by imaging soon after transfer to normoxia and 24 and $72 \mathrm{hrs}$, as described under methods. The addition of ADMA inhibited both iCM contractility velocity and rate recovery, which was rescued in the presence of rDDAH-1. The sample size for all groups was $n=3$. All data are expressed as mean $\pm S D$. Significance determined by ADMA-compared one-way ANOVA with Dunnett's post hoc test. ${ }^{*} \mathrm{p}<0.05,{ }^{* *} \mathrm{p}<0.01$, ${ }^{* * *} \mathrm{p}<0.001$.

Figure 5. rDDAH-1 improved ADMA-induced mitochondrial respiration capacity in adult male mouse cardiomyocytes. A. Shows Seahorse Cell Mito Stress Test assay in control cardiomyocytes from adult male mouse hearts and the calculable parameters. Dotted lines indicate addition of ATP synthase inhibitor oligomycin, electron transport chain uncoupler FCCP and complex I and III blockers rotenone and antimycin A. B. Oxygen consumption rate $(\mathrm{OCR})$ in mouse primary cardiomyocytes treated with 2 hrs vehicle (Control), ADMA (3 $\mu \mathrm{M})$, or ADMA + rDDAH-1 $(20 \mu \mathrm{g} / \mathrm{mL})$, respectively. C-F. Calculated values for respiratory parameters. The experiment was performed on cardiomyocytes from a total of three male mouse hearts. All data are given as mean \pm SD. Significance determined by unpaired t-test. ${ }^{*} p<0.05,{ }^{* *} p<0.01$.

Figure 6. rDDAH-1 reduced ADMA induced ROS generation in cardiomyocytes. Mitochondrial superoxide production in mouse cardiomyocytes was determined using MitoSOX Red. A. Representative images in cardiomyocytes with MitoSOX Red. B. 
Concentration-dependent changes ROS after 2 hrs treatment with $\operatorname{ADMA}(0,3,10$, and $30 \mu \mathrm{M}$. The data are ${ }^{\star \star * *} \mathrm{p}<0.0001$, Kruskal-Wallis test with Dunn's comparison. C.

rDDAH-1 $(20 \mu \mathrm{g} / \mathrm{mL})$ treatment decreased mitochondrial superoxide production in mouse cardiomyocytes. The data are ${ }^{*} p<0.05,{ }^{* * *} p<0.0001$, Mann-Whitney test. The experiment was repeated in two individual trials and cardiomyocytes were isolated from a total of three male mouse hearts. All data are given as mean \pm SD.

Figure 7. rDDAH-1 modified cytokine expression following $\mathrm{I} / \mathrm{R}$ in mouse heart. The perfusate from the mouse heart I/R study were collected at the end of the experiment and concentrarted 10 -fold by membrane centrifugation. A. The concentration of each cytokine was determined in the samples from the effluent of mouse hearts without $I / R$ (baseline), with I/R but no rDDAH-1 treatment (vehicle) and with I/R plus DDAH treatment $(\mathrm{rDDAH}-1)$. The level of each cytokine among the three groups was compared, and those with $p$ values grerater than 0.05 are listed as not significant (NS). B. Shows data on key cytokine expression (IL-9, IL-10, IL-13, IL-17A) in the perfusate at baseline, after I/R (vehicle), and I/R with $0.1 \mu \mathrm{g} / \mathrm{mL} \mathrm{rDDAH}-1$ treatment. All data are given as mean \pm SD. Significance determined by baseline-compared one-way ANOVA with Dunnett's post hoc test or vehicle-compared unpaired t-test. ${ }^{*} \mathrm{p}<0.05,{ }^{* *} \mathrm{p}<0.01$, ${ }^{* * *} p<0.001$

Figure 8. ADMA and rDDAH-1 modulate IL-17A expression in cardiac and endothelial cells. A. A summary of key cytokine changes $(p<0.05)$ in response to ADMA and rDDAH-1 in various cells in vitro. Figures B-E. show changes IL-17A in 
mouse heart effluent (B), human iCM (C), mouse cardiomyocyte (CM) (D), HUVEC (E) in the presence or absence of ADMA or ADMA+rDDAH-1. All data are expressed as mean \pm SD. Significance determined by baseline- or control-compared one-way ANOVA with Dunnett's post hoc test or vehicle- or ADMA-compared unpaired t-test. ${ }^{*} \mathrm{p}<0.05$, ${ }^{* *} \mathrm{p}<0.01,{ }^{* * *} \mathrm{p}<0.001$. 
A

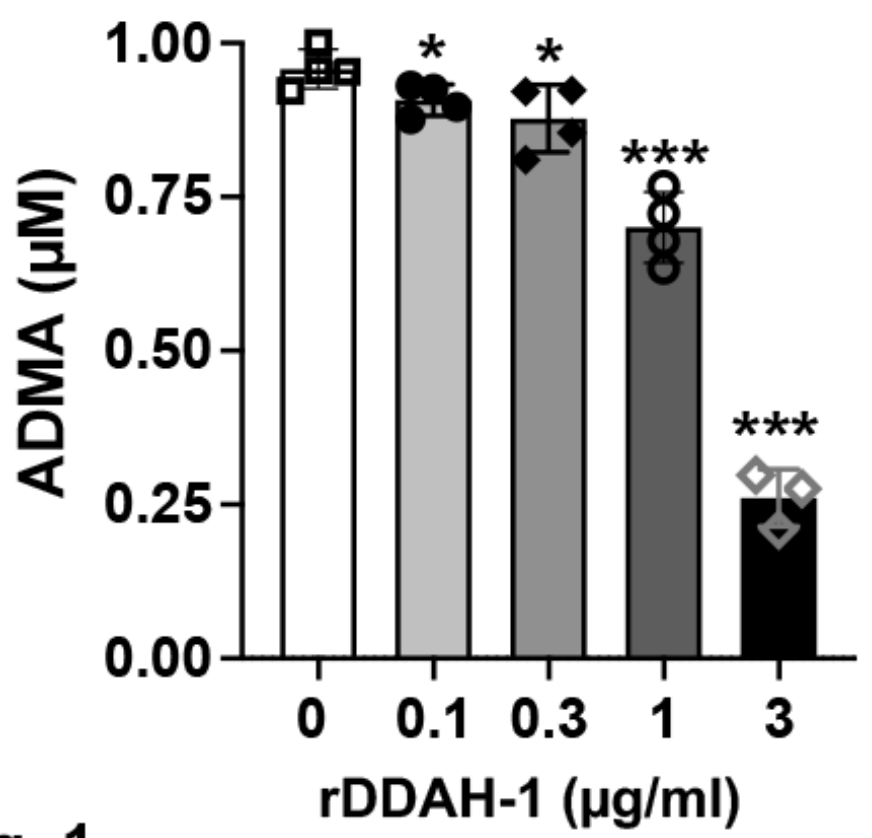

B

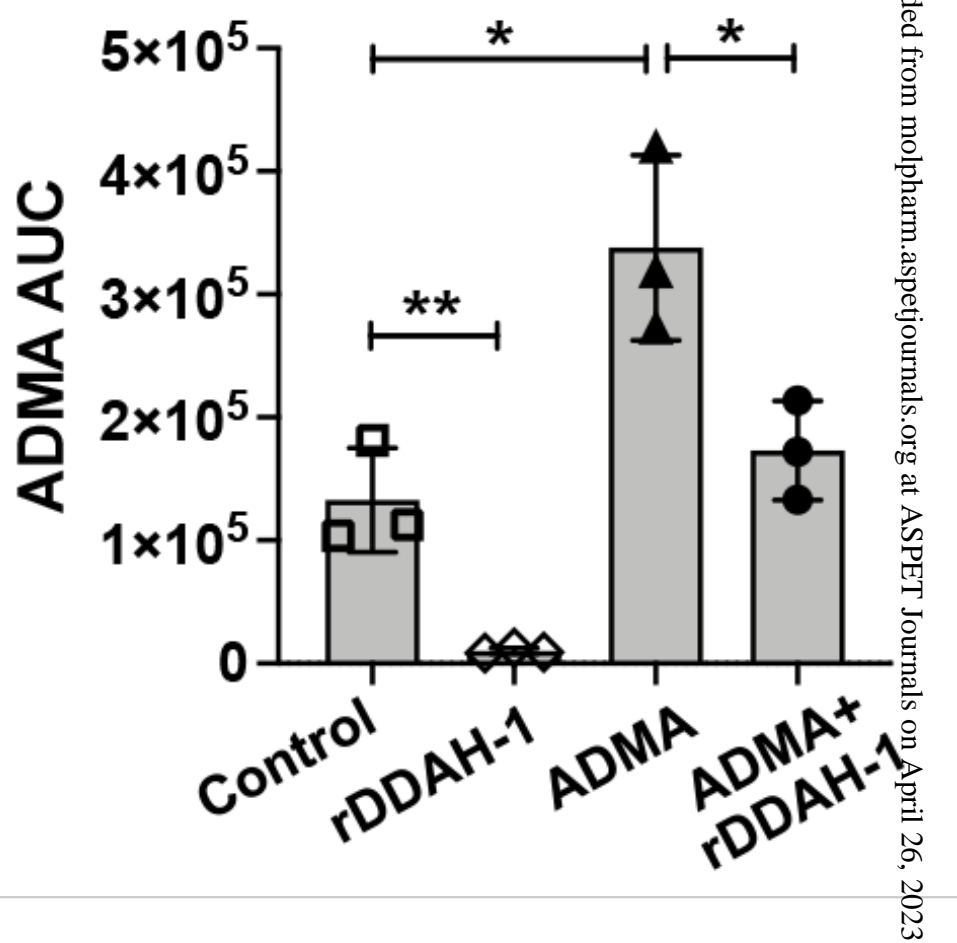


A

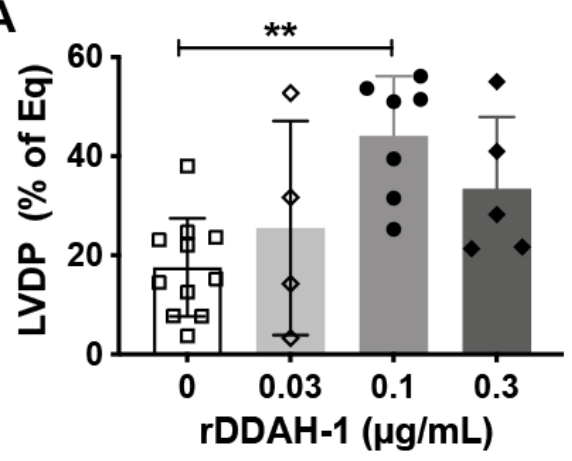

D

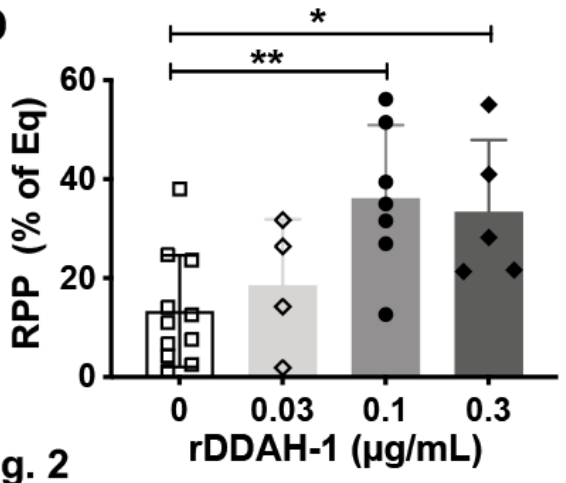

B

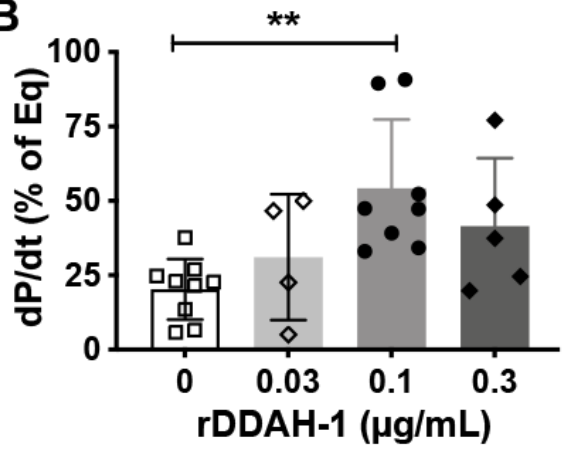

E

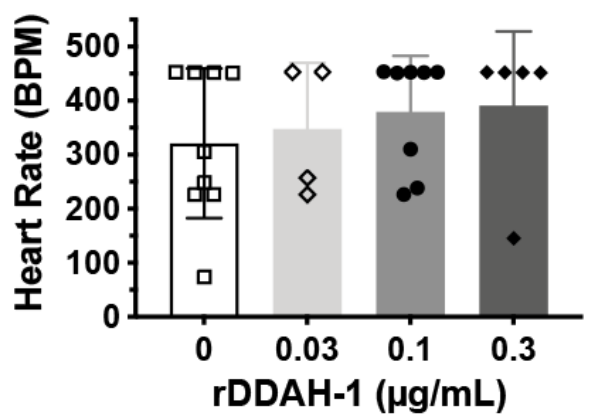

C

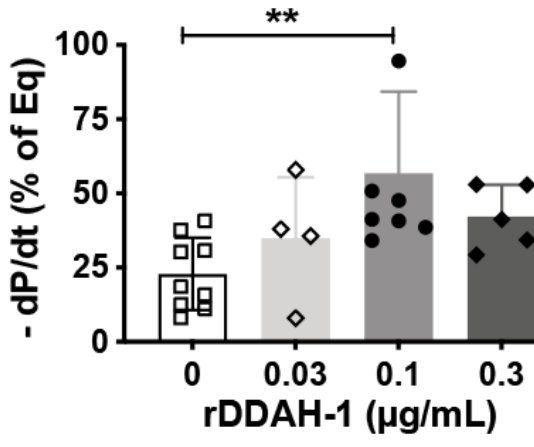

$\mathbf{F}$

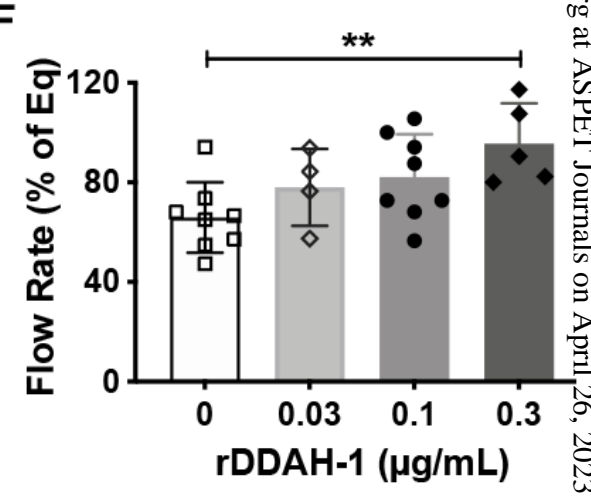


A

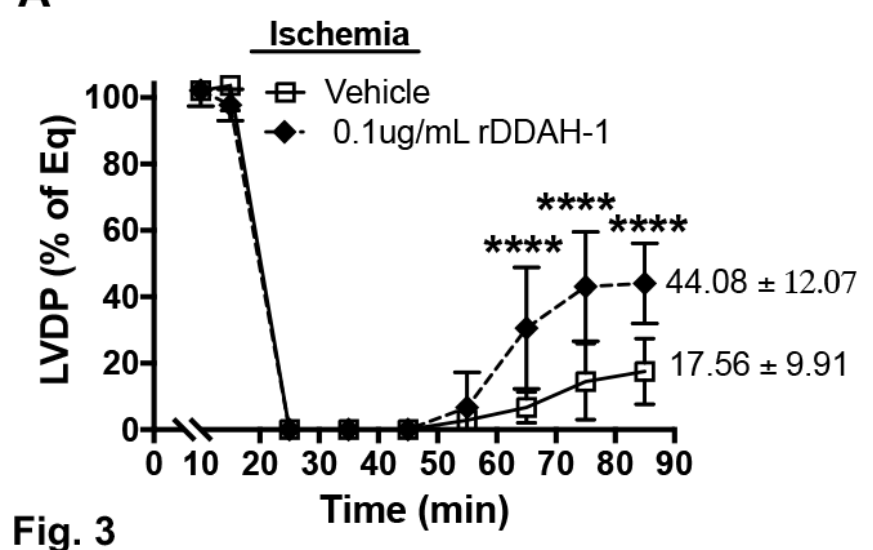

B

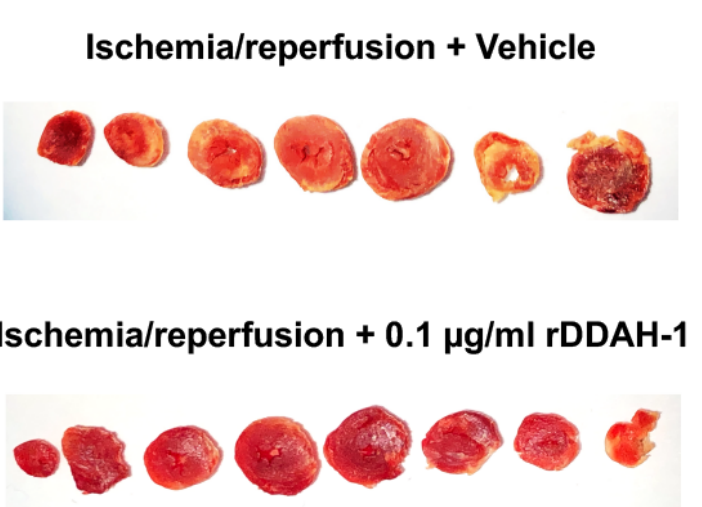

C

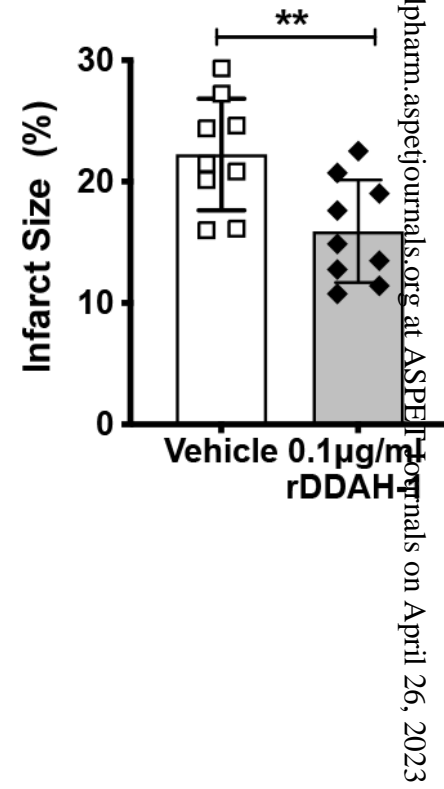


A

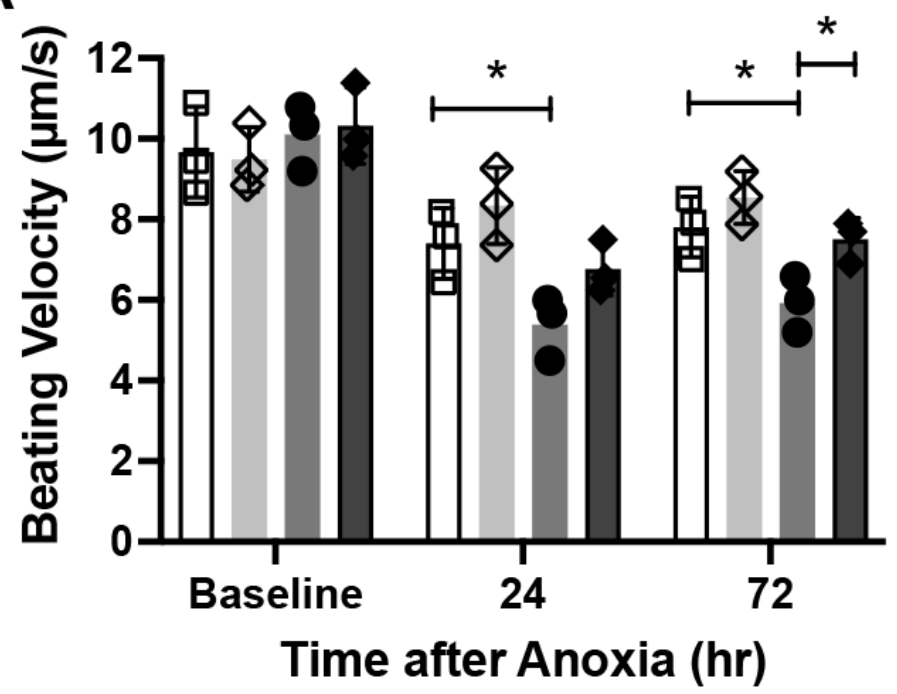

B

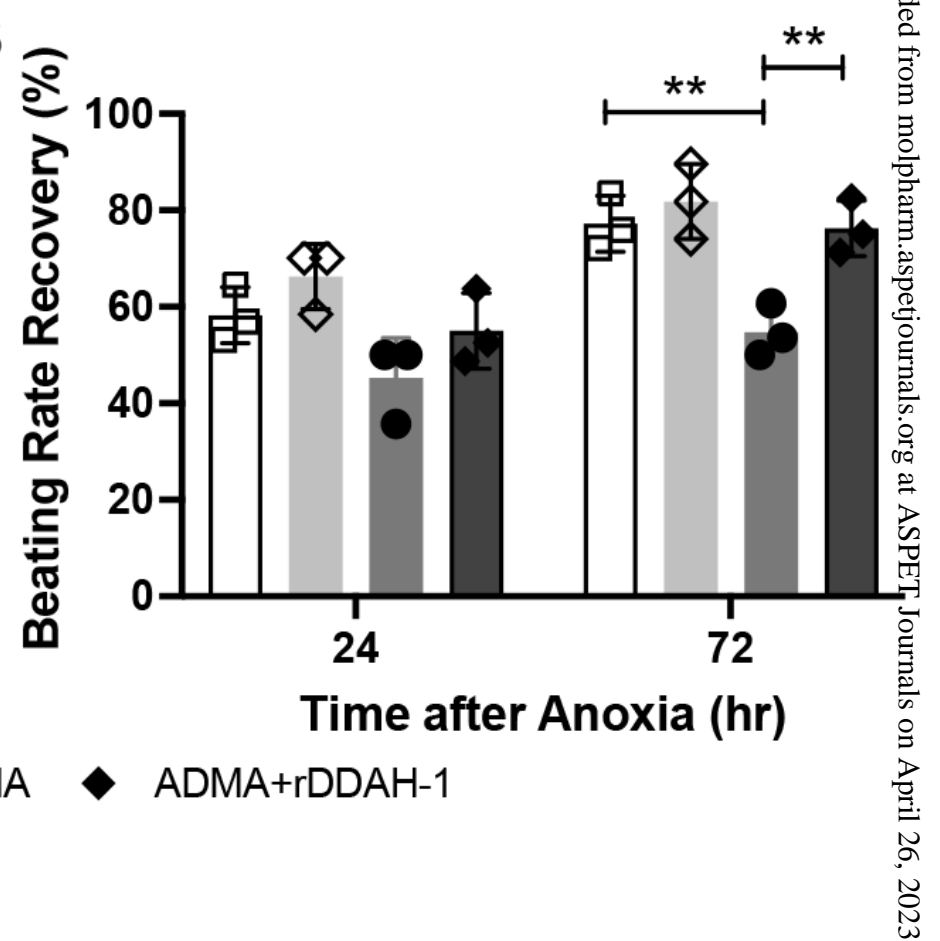

Fig. 4

$\square$ Control $\diamond \mathrm{rDDAH}-1$

ADMA 
A

Mitochondrial Respiration

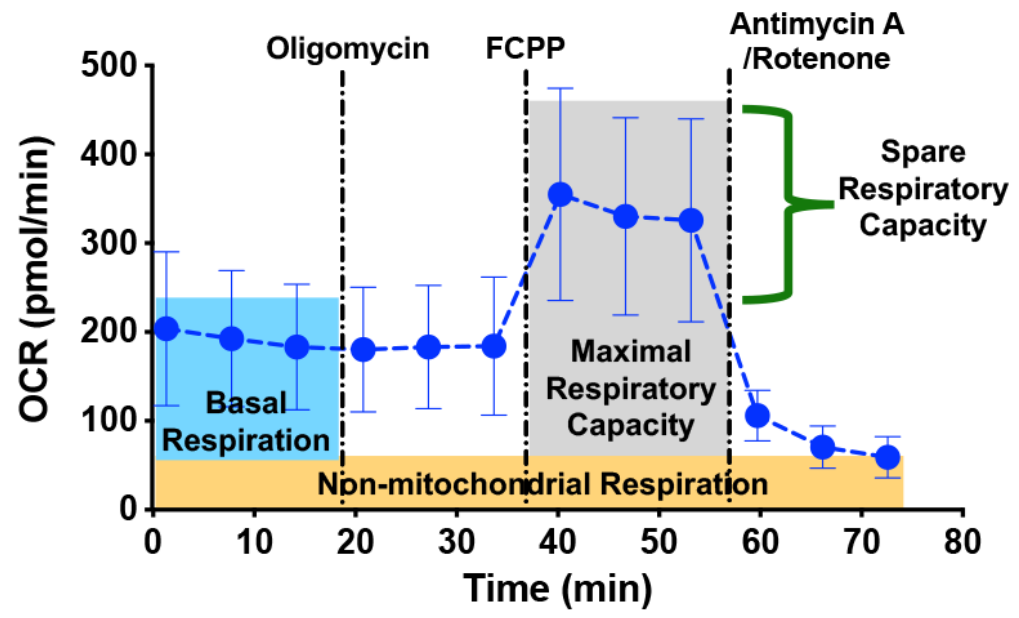

C

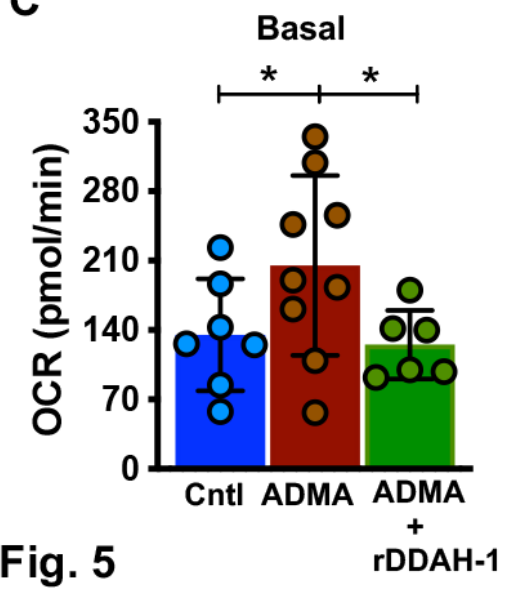

B

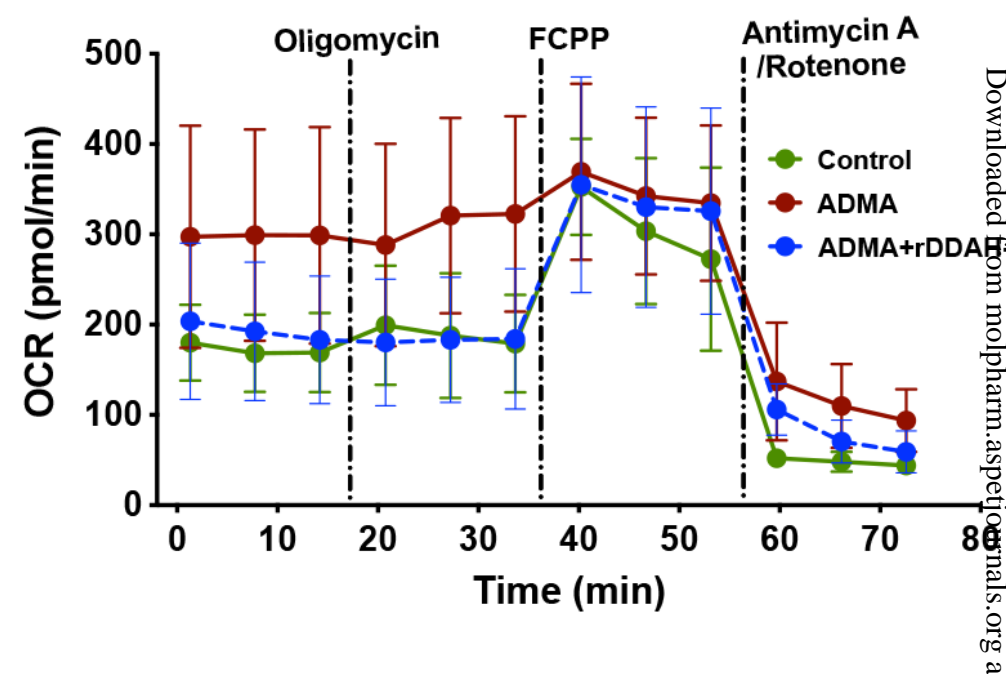

E

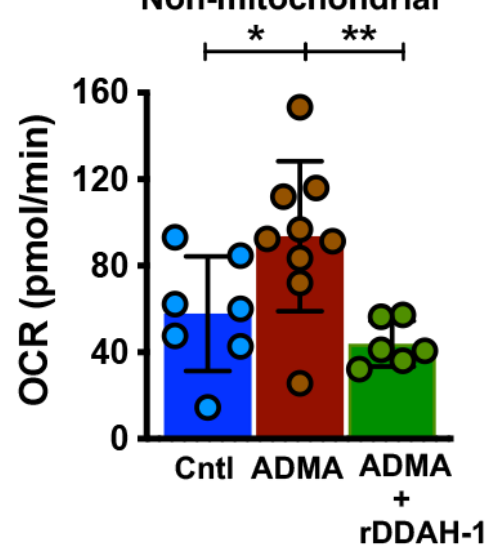

Maximal

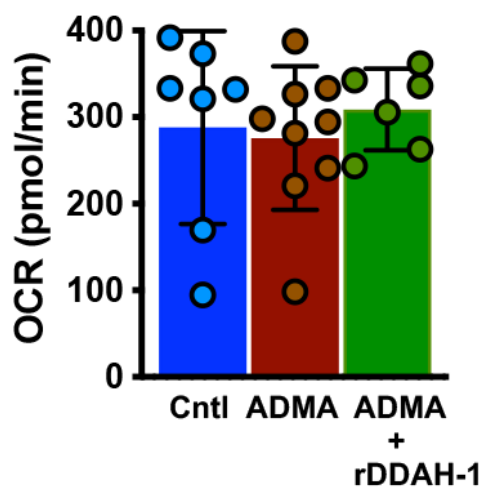

$\mathbf{F}$

Spare Respiratory Capacity

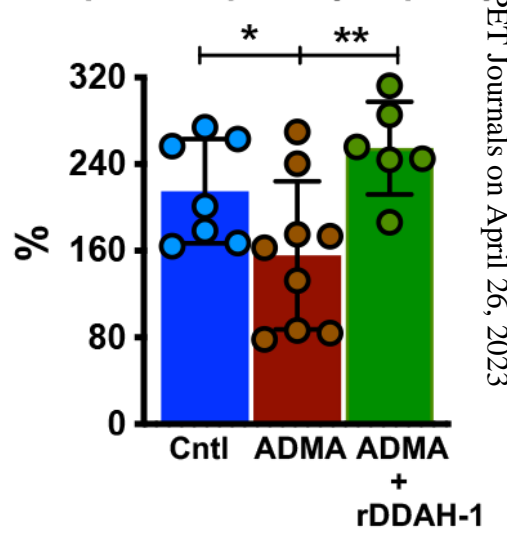


A

\section{Phase Contrast}

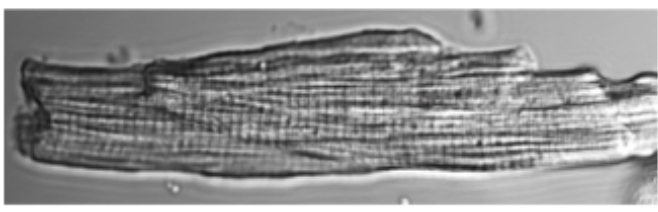

MitoSOX Red

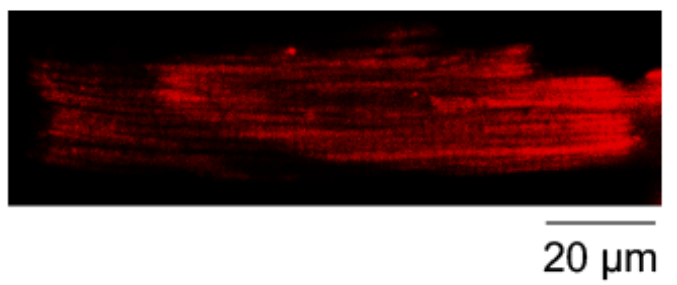

C

Fig. 6
4

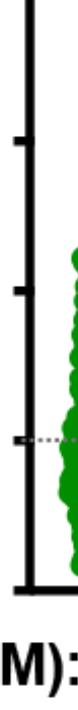

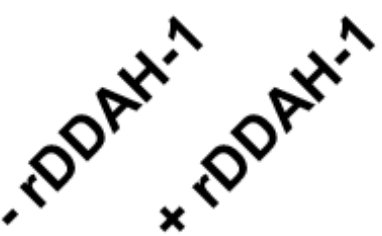

B

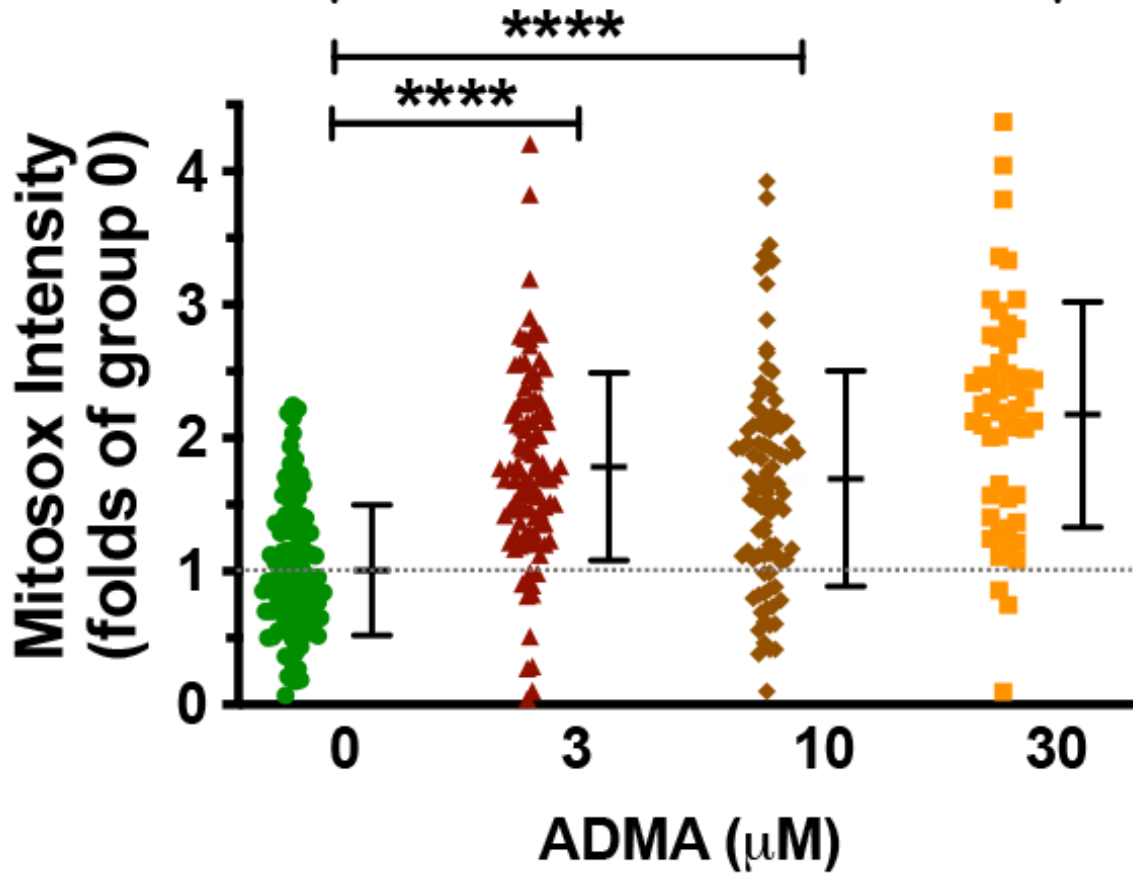

$* * * *$

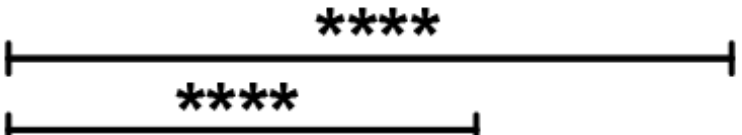

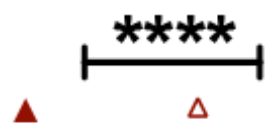

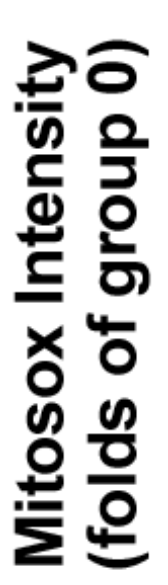

$\boldsymbol{\Delta}$

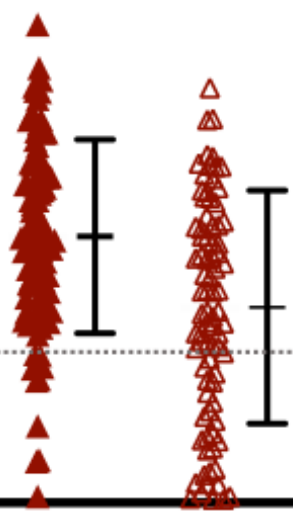

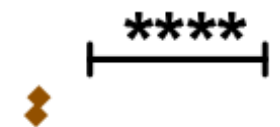

8

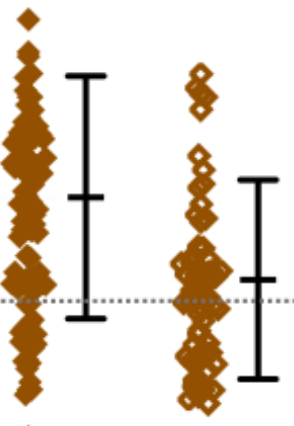

ADMA $(\mu \mathrm{M}): \quad 0$

10

30

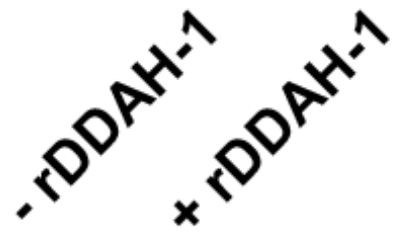

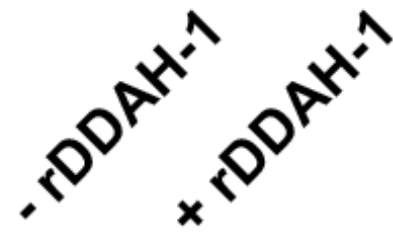

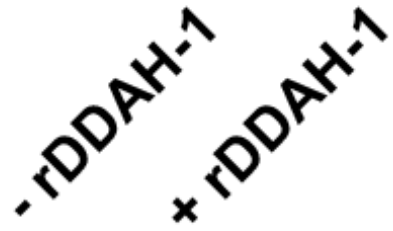




\begin{tabular}{|c|c|c|}
\hline Cytokine & $\begin{array}{c}\text { Baseline vs } \\
\text { rDDAH-1 }\end{array}$ & $\begin{array}{c}\text { Vehicle vs } \\
\text { rDDAH-1 }\end{array}$ \\
\hline CCL2 & NS & NS \\
\hline CCL5 & NS & NS \\
\hline CSF1 & NS & NS \\
\hline CSF2 & NS & NS \\
\hline CXCL1 & NS & NS \\
\hline IFN-g & NS & NS \\
\hline TNF-a & NS & NS \\
\hline VEGFA & NS & NS \\
\hline IL1A & NS & NS \\
\hline IL1B & NS & NS \\
\hline IL2 & NS & NS \\
\hline IL3 & NS & NS \\
\hline IL4 & NS & NS \\
\hline IL5 & NS & NS \\
\hline IL6 & NS & NS \\
\hline IL9 & NS & P $<0.05$ \\
\hline IL10 & NS & P $<0.01$ \\
\hline IL12A & NS & NS \\
\hline IL13 & P $<0.05$ & P $<0.01$ \\
\hline IL17A & P $<0.01 ~$ & P $<0.001$ \\
\hline IL & & \\
\hline
\end{tabular}

B
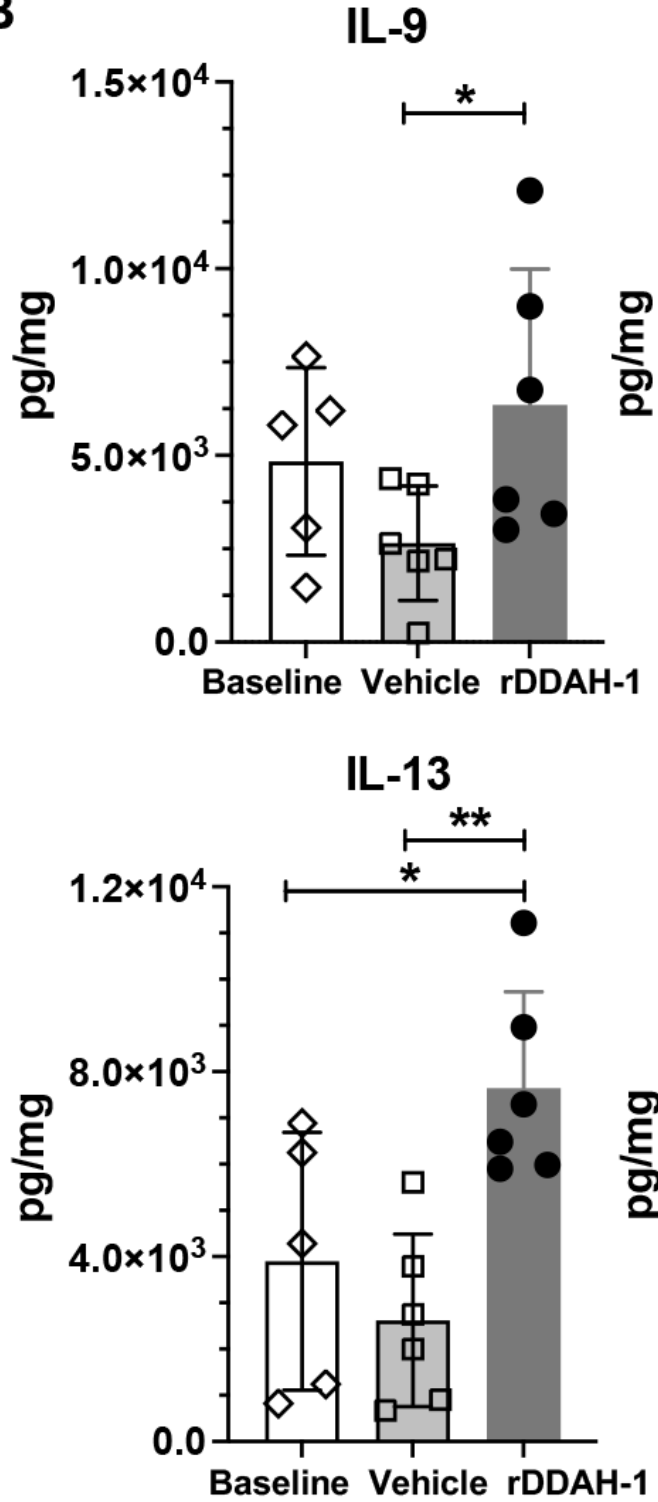

IL-10

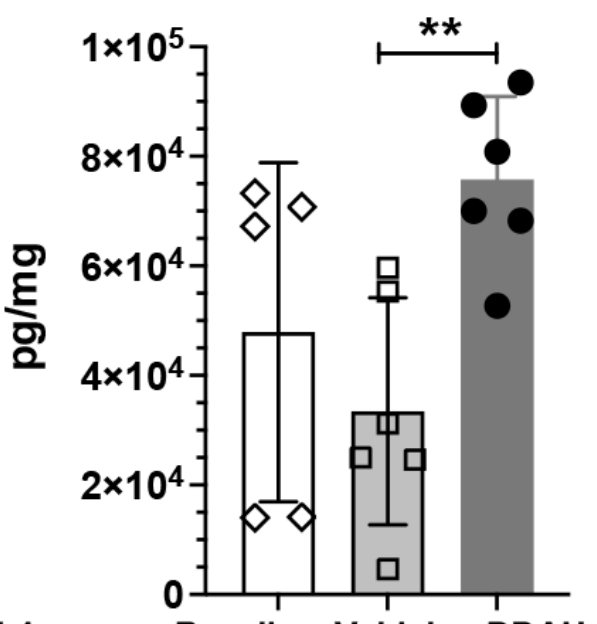

Baseline Vehicle rDDAH-1

IL-17A

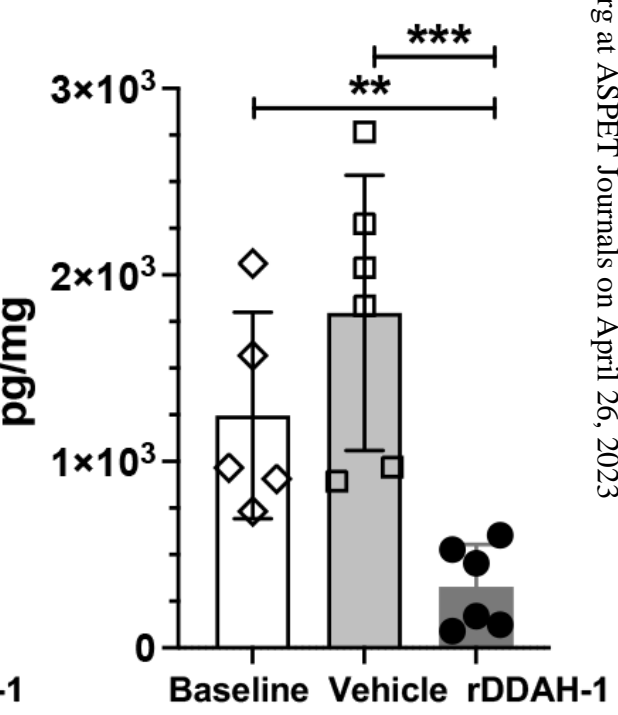

Fig. 7 


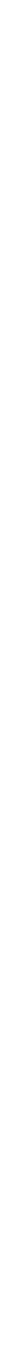

